\title{
SLICE REGULAR COMPOSITION OPERATORS
}

\author{
GUANGBIN REN AND XIEPING WANG
}

\begin{abstract}
In the article the class of slice regular functions is shown to be closed under a new regular composition. The new regular composition turns out to be globally defined in contrast to the locally defined version by Vlacci. Its advantage over Vlacci's is demonstrated by its associated theory of composition operators and dynamical systems for slice regular functions. Especially, the corresponding Littlewood subordination principle and the Denjoy-Wolff type theorem can be established.
\end{abstract}

\section{INTRODUCTION}

It is known that the composition $f(\varphi(x))$ of any two formal power series

$$
f(x)=\sum_{j=0}^{\infty} x^{j} a_{j}, \quad \varphi(x)=\sum_{k=0}^{\infty} x^{k} b_{k}
$$

is a formal power series when coefficients $a_{j}, b_{k}$ are taken in a commutative field and $b_{0}=0$. However, if the constant term $b_{0}$ of the power series $\varphi$ is not 0 , the existence of the composition $f(\varphi(x))$ has been an open problem for many years. Only recently has received some partial answers [3, 11] and these results are also extended to the noncommutative setting by Vlacci [26] for slice regular functions.

In this article a new slice regular composition of any two slice regular functions will be introduced based on slice regular product. It has the advantage over Vlacci's that the new composition can thus lead to the theory of composition operators for slice regular functions.

The theory of slice regular functions is initiated by Gentili and Struppa 16, 17. It has elegant applications in the functional calculus for noncommutative operators 7]. The detailed up-to-date theory appears in the monograph [14. The theory of slice regular functions is associated with the non-elliptic differential operator with nonconstant coefficients given by

$$
|\underline{x}|^{2} \frac{\partial}{\partial x_{0}}+\underline{x} \sum_{j=1}^{3} x_{j} \frac{\partial}{\partial x_{j}}
$$

where $\underline{x}$ is the vector part of the quaternion $x=x_{0}+\underline{x} \in \mathbb{H}$, see $[\underline{6}$ for more details.

We now introduce the slice regular composition operator $C_{\varphi}$ on the slice regular Hardy space $H^{2}(\mathbb{B})$. Let $\varphi: \mathbb{B} \rightarrow \mathbb{B}$ be a slice regular function on the open unit

2010 Mathematics Subject Classification. 30G35, 32A26.

Key words and phrases. Slice regular functions, Regular composition, Denjoy-Wolff type theorem, Littlewood subordination principle, Composition operators.

This work was supported by the NNSF of China (11071230), RFDP (20123402110068). 
ball $\mathbb{B}$ of quaternions and we define

$$
C_{\varphi}(f)(q)=\sum_{n=0}^{\infty} \varphi^{* n}(q) a_{n},
$$

where the power series expansion of $f \in H^{2}(\mathbb{B})$ takes the form

$$
f(q)=\sum_{n=0}^{\infty} q^{n} a_{n}
$$

and $\varphi^{* n}$ is the $n$th regular power of $\varphi$ with respect to $*$-product.

It is worth remarking here that $C_{\varphi} f$ is well defined and slice regular in $\mathbb{B}$ globally (see Section 4 below for details) while the Vlacci's version of slice regular composition is, in general, only defined in $\frac{1}{3} \mathbb{B}$ other than the whole ball $\mathbb{B}$, due to the Bohr phenomenon, see Section 3 for details.

Our first main result is the Littlewood subordination principle for slice regular composition.

Theorem 1.1. (Littlewood Subordination Principle) Let $\varphi: \mathbb{B} \rightarrow \mathbb{B}$ be a slice regular function and $\varphi(0)=0$, then $C_{\varphi}$ is a bounded composition operator on $H^{2}(\mathbb{B})$ with norm $\left\|C_{\varphi}\right\|=1$.

We remark that, without the restriction $\varphi(0)=0$, the exact norm of a composition operator $C_{\varphi}$ is still unknown even in the classical holomorphic Hardy space $H^{2}(\mathbb{D})$. In the present article, we can provide the exact norm of a composition operator on the slice regular Hardy space $H^{2}(\mathbb{B})$ in the special case of which is induced by the slice regular Möbius transformations. We refer to [8, 2, 10, for related questions concerning the norms of holomorphic composition operators.

Theorem 1.2. Let $\varphi$ be a regular Möbius transformation of $\mathbb{B}$, then the composition operators $C_{\varphi}$ is bounded on $H^{2}(\mathbb{B})$ with norm

$$
\left\|C_{\varphi}\right\|=\left(\frac{1+|\varphi(0)|}{1-|\varphi(0)|}\right)^{\frac{1}{2}}
$$

When $\varphi(\mathbb{B}) \subset \subset \mathbb{B}$, we know that the composition operator is compact on regular Hardy space $H^{p}(\mathbb{B})$.

Theorem 1.3. Let $\varphi: \mathbb{B} \rightarrow \mathbb{B}$ be a regular function on $\mathbb{B}$ such that $\|\varphi\|_{\infty}<1$. Then $C_{\varphi}$ is a compact operator on $H^{p}(\mathbb{B})$ with $1 \leq p \leq \infty$.

The feature of our new slice regular composition lies at its analogous dynamical behaviors of the iterates of slice regular self-mappings as in the holomorphic setting. In particular, we have the Denjoy-Wolff type theorem for slice regular mappings.

Theorem 1.4. (Denjoy-Wolff) Let $I d \neq f \in A u t(\mathbb{B})$ be not elliptic automorphism in which $f\left(\mathbb{B}_{I}\right) \subseteq \mathbb{B}_{I}$ for some $I \in \mathbb{S}$, then the sequence of $\left\{f^{\odot n}\right\}$ uniformly converges on every compact subset of $\mathbb{B}$ to a boundary fixed point of $f$.

The paper consists of eight sections besides the introduction, and its outline is as follows. In Section 2, we set up the basic notations and give the preliminary results. Section 3 is devoted to recalling the Vlacci's regular composition for slice regular functions. In Section 4, we define our new regular composition and investigate its basic properties. In Section 5, we will investigate some results about the dynamical behaviors of the iterates of slice regular self-mappings $f$ of $\mathbb{B}$ under the 
extra assumption that $f$ preserves at least one slice. The assumption is satisfied for the slice regular automorphism group and all the elementary functions in the slice regular theory. In Section 6, we establish the Littlewood subordination principle with respect to our new regular composition. The boundedness and compactness of composition operators are considered in Section 7 and Section 8, respectively. Finally, Section 9 comes the conclusion of the paper.

\section{Preliminaries}

We recall in this section some preliminary definitions and results on slice regular functions. Let $\mathbb{H}$ denote the noncommutative, associative, real algebra of quaternions with standard basis $\{1, i, j, k\}$, subject to the multiplication rules

$$
i^{2}=j^{2}=k^{2}=i j k=-1 \text {. }
$$

Every element $q=x_{0}+x_{1} i+x_{2} j+x_{3} k$ in $\mathbb{H}$ is composed by the real part $\operatorname{Re}(q)=x_{0}$ and the imaginary part $\operatorname{Im}(q)=x_{1} i+x_{2} j+x_{3} k$. The conjugate of $q \in \mathbb{H}$ is then $\bar{q}=\operatorname{Re}(q)-\operatorname{Im}(q)$ and its modulus is defined by $|q|^{2}=q \bar{q}=|\operatorname{Re}(q)|^{2}+|\operatorname{Im}(q)|^{2}$. We can therefore calculate the multiplicative inverse of each $q \neq 0$ as $q^{-1}=|q|^{-2} \bar{q}$. Every $q \in \mathbb{H}$ can be expressed as $q=x+y I$, where $x, y \in \mathbb{R}$ and

$$
I=\frac{\operatorname{Im}(q)}{|\operatorname{Im}(q)|}
$$

if $\operatorname{Im} q \neq 0$, otherwise we take $I$ arbitrarily such that $I^{2}=-1$. Then $I$ is an element of the unit 2 -sphere of purely imaginary quaternions,

$$
\mathbb{S}=\left\{q \in \mathbb{H}: q^{2}=-1\right\} .
$$

For every $I \in \mathbb{S}$, we denote by $\mathbb{C}_{I}$ the plane $\mathbb{R} \oplus I \mathbb{R}$, isomorphic to $\mathbb{C}$, and, if $\Omega \subseteq \mathbb{H}$, by $\Omega_{I}$ the intersection $\Omega \cap \mathbb{C}_{I}$. Also, for $R>0$, we will denote the open ball centred at the origin with radius $R$ by

$$
B(0, R)=\{q \in \mathbb{H}:|q|<R\} .
$$

We can now recall the definition of slice regularity.

Definition 2.1. Let $\Omega$ be a domain in $\mathbb{H}$. A function $f: \Omega \rightarrow \mathbb{H}$ is called slice regular if, for all $I \in \mathbb{S}$, its restriction $f_{I}$ to $\Omega_{I}$ is holomorphic, i.e. it has continuous partial derivatives and satisfies

$$
\bar{\partial}_{I} f(x+y I):=\frac{1}{2}\left(\frac{\partial}{\partial x}+I \frac{\partial}{\partial y}\right) f_{I}(x+y I)=0
$$

for all $x+y I \in \Omega_{I}$.

As shown in 4, a class of domains, the so-called symmetric slice domains naturally qualify as domains of definition of slice regular functions.

Definition 2.2. Let $\Omega$ be a domain in $\mathbb{H}$. $\Omega$ is called a slice domain if $\Omega$ intersects the real axis and $\Omega_{I}$ is a domain of $\mathbb{C}_{I}$ for any $I \in \mathbb{S}$.

Moreover, if $x+y I \in \Omega$ implies $x+y \mathbb{S} \subseteq \Omega$ for any $x, y \in \mathbb{R}$ and $I \in \mathbb{S}$, then $\Omega$ is called a symmetric slice domain.

From now on, we will omit the term 'slice' when referring to slice regular functions. A natural notion of derivative can be given for regular functions as follows (see [16, 17]). 
Definition 2.3. Let $\Omega$ be a slice domain in $\mathbb{H}$, and let $f: \Omega \rightarrow \mathbb{H}$ be a regular function. The slice derivative of $f$ at $q=x+y I$ is defined by

$$
\partial_{I} f(x+y I):=\frac{1}{2}\left(\frac{\partial}{\partial x}-I \frac{\partial}{\partial y}\right) f_{I}(x+y I) .
$$

Notice that the operators $\partial_{I}$ and $\bar{\partial}_{I}$ commute, and $\partial_{I} f=\frac{\partial f}{\partial x}$ for regular functions. Therefore, the slice derivative of a regular function is still regular so that we can iterate the differentiation to obtain the $n$-th slice derivative

$$
\partial_{I}^{n} f=\frac{\partial^{n} f}{\partial x^{n}}, \quad \forall n \in \mathbb{N} .
$$

In what follows, for the sake of simplicity, we will directly denote the $n$-th slice derivative $\partial_{I}^{n} f$ by $f^{(n)}$ for every $n \in \mathbb{N}$.

As stated in [17, a quaternionic power series $\sum_{n=0}^{\infty} q^{n} a_{n}$ with $\left\{a_{n}\right\}_{n \in \mathbb{N}} \subset \mathbb{H}$ defines a regular function in its domain of convergence, which proves to be an open ball $B(0, R)$ with $R$ equal to the radius of convergence of the power series. The converse result is also true.

Theorem 2.4. (Taylor Expansion) A function $f$ is regular on $B=B(0, R)$ if and only if $f$ has a power series expansion

$$
f(q)=\sum_{n=0}^{\infty} q^{n} a_{n} \quad \text { with } \quad a_{n}=\frac{f^{(n)}(0)}{n !} .
$$

We can recover the values of a regular function on a symmetric slice domain from its values on a single slice $\mathbb{C}_{I}$, due to the following representation formula (a special case of a result in [4]), which was proven in [5.

Theorem 2.5. (Representation Formula) Let $f$ be a regular function on a symmetric slice domain $\Omega \subseteq \mathbb{H}$ and let $I \in \mathbb{S}$. Then for all $x+y J \in \Omega$ with $J \in \mathbb{S}$, the following equality holds

$$
f(x+y J)=\frac{1}{2}(f(x+y I)+f(x-y I))+\frac{1}{2} J I(f(x-y I)-f(x+y I)) .
$$

A fundamental result in the theory of regular functions is described by the splitting lemma (see [17]), which relates slice regularity to classical holomorphy.

Lemma 2.6. (Splitting Lemma) Let $f$ be a regular function on a slice domain $\Omega \subseteq \mathbb{H}$. Then for any $I \in \mathbb{S}$ and any $J \in \mathbb{S}$ with $J \perp I$, there exist two holomorphic functions $F, G: \Omega_{I} \rightarrow \mathbb{C}_{I}$ such that for every $z=x+y I \in \Omega_{I}$, the following equality holds

$$
f_{I}(z)=F(z)+G(z) J .
$$

The pointwise product of two regular functions is not, in general, regular. To maintain the regularity, a new multiplication operation, the $*$-product, was introduced. On open balls centered at the origin, the $*$-product of two regular functions can be defined by means of their power series expansions in [12, mimicking the standard multiplication of polynomials in a skew field. However, the generalization to the symmetric slice domains is based on the extension lemma (see [4]). 
Lemma 2.7. (Extension Lemma) Let $\Omega$ be a symmetric slice domain and choose $I \in \mathbb{S}$. If $f_{I}: \Omega_{I} \rightarrow \mathbb{H}$ is holomorphic, then setting

$$
f(x+y J)=\frac{1}{2}\left(f_{I}(x+y I)+f_{I}(x-y I)\right)+\frac{1}{2} J I\left(f_{I}(x-y I)-f_{I}(x+y I)\right)
$$

extends $f_{I}$ to a regular function $f: \Omega \rightarrow \mathbb{H}$. Moreover, $f$ is the unique extension and it is denoted by $\operatorname{ext}\left(f_{I}\right)$.

In order to define the regular product of $f$ and $g$, which are regular functions on a symmetric slice domain $\Omega \subseteq \mathbb{H}$, we apply the splitting lemma to write

$$
f_{I}(z)=F(z)+G(z) J, \quad g_{I}(z)=H(z)+K(z) J
$$

where $F, G, H, K: \Omega_{I} \rightarrow \mathbb{C}_{I}$ are holomorphic functions and $I, J \in \mathbb{S}$ with $I \perp J$.

Let $f_{I} * g_{I}: \Omega_{I} \rightarrow \mathbb{C}_{I}$ be the holomorphic function defined by

$$
f_{I} * g_{I}(z)=(F(z) H(z)-G(z) \overline{K(\bar{z})})+(F(z) K(z)+G(z) \overline{H(\bar{z})}) J .
$$

The regular extension of $f_{I} * g_{I}(z)$ is defined to be the regular product (see [4]).

Definition 2.8. Let $f, g$ be two regular functions on a symmetric slice domain $\Omega \subseteq \mathbb{H}$. The regular product (or $*$-product) of $f$ and $g$ is the function defined by

$$
f * g(q)=\operatorname{ext}\left(f_{I} * g_{I}\right)(q)
$$

regular on $\Omega$.

Notice that the $*$-product is associative and is not, in general, commutative. It can be described in terms of the usual pointwise product (see [4, 12]):

Proposition 2.9. Let $f$ and $g$ be two regular functions on a symmetric slice domain $\Omega \subseteq \mathbb{H}$. Then for all $q \in \Omega$,

$$
f * g(q)=\left\{\begin{array}{cll}
f(q) g\left(f(q)^{-1} q f(q)\right) & \text { if } & f(q) \neq 0 \\
0 & \text { if } & f(q)=0 .
\end{array}\right.
$$

Corollary 2.10. Let $f$ and $g$ be two regular functions on a symmetric slice domain $\Omega \subseteq \mathbb{H}$ and let $q \in \mathbb{H}$. Then $f * g(q)=0$ if and only if $f(q)=0$ or $f(q) \neq$ 0 and $g\left(f(q)^{-1} q f(q)\right)=0$.

The theory of zero set of regular functions depends heavily on its conjugation and symmetrization.

Definition 2.11. Let $f$ be a regular function on a symmetric slice domain $\Omega \subseteq \mathbb{H}$ and suppose that

$$
f_{I}(z)=F(z)+G(z) J
$$

is the splitting of $f$, where $I, J \in \mathbb{S}$ and $I \perp J$.

Consider the holomorphic function

$$
f_{I}^{c}(z)=\overline{F(\bar{z})}-G(z) J .
$$

The regular conjugate of $f$ is the function defined by

$$
f^{c}(q)=\operatorname{ext}\left(f_{I}^{c}\right)(q) .
$$

The symmetrization of $f$ is the function defined by

$$
f^{s}(q)=f * f^{c}(q)=f^{c} * f(q) .
$$

Both $f^{c}$ and $f^{s}$ are regular functions on $\Omega$. 
The relation among the zero sets of $f, f^{c}$ and $f^{s}$ has been fully characterized (see [12, 13]):

Proposition 2.12. Let $f$ be a regular function on a symmetric slice domain $\Omega$. For all $x, y \in \mathbb{R}$ with $x+y \mathbb{S} \subseteq \Omega$, the zeros of the regular conjugate $f^{c}$ on $x+y \mathbb{S}$ are in one-to-one correspondence with those of $f$. Moreover, the symmetrization $f^{s}$ vanishes exactly on the sets $x+y \mathbb{S}$ on which $f$ has a zero.

Similarly, there is a close relation between the norms of $f$ and $f^{c}$, when restricted to a sphere $x+y \mathbb{S}$ (see [23]):

Proposition 2.13. Let $f$ be a regular function on the unit ball $\mathbb{B}=B(0,1)$. For any sphere of the form $x+y \mathbb{S}$ contained in $\mathbb{B}$, the following equalities hold true

$$
\sup _{I \in \mathbb{S}}|f(x+y I)|=\sup _{I \in \mathbb{S}}\left|f^{c}(x+y I)\right|, \quad \inf _{I \in \mathbb{S}}|f(x+y I)|=\inf _{I \in \mathbb{S}}\left|f^{c}(x+y I)\right| .
$$

We now recall more results from [14].

Theorem 2.14. (Identity Principle) Let $f$ be a regular function on a slice domain $\Omega \subseteq \mathbb{H}$. Denote by $\mathcal{Z}_{f}$ the zero set of $f$,

$$
\mathcal{Z}_{f}=\{q \in \Omega: f(q)=0\} .
$$

If there exists an $I \in \mathbb{S}$ such that $\Omega_{I} \cap \mathcal{Z}_{f}$ has an accumulation point in $\Omega_{I}$, then $f$ vanishes identically on $\Omega$.

Theorem 2.15. (Maximum Modulus Principle) Let $f$ be a regular function on a slice domain $\Omega \subseteq \mathbb{H}$. If $|f|$ has a relative maximum in $\Omega$, then $f$ is constant in $\Omega$.

Theorem 2.16. (Schwarz Lemma) Let $f: \mathbb{B} \rightarrow \mathbb{B}$ be a regular function. If $f(0)=0$, then

$$
|f(q)| \leq|q|
$$

for all $q \in \mathbb{B}$ and

$$
\left|f^{\prime}(0)\right| \leq 1
$$

Both inequalities are strict (except at $q=0$ ) unless $f(q)=q u$ for some $u \in \partial \mathbb{B}$.

The quaternionic counterpart of complex Hardy spaces was considered in 9 .

Definition 2.17. Let $f$ be a regular function on $\mathbb{B}$ and let $0<p<\infty$. Set

$$
\|f\|_{p}=\sup _{I \in \mathbb{S}} \lim _{r \rightarrow 1^{-}}\left(\frac{1}{2 \pi} \int_{-\pi}^{\pi}\left|f\left(r e^{I \theta}\right)\right|^{p} d \theta\right)^{\frac{1}{p}}
$$

and

$$
\|f\|_{\infty}=\sup _{q \in \mathbb{B}}|f(q)| .
$$

Then for any $0<p \leq \infty$, the slice regular Hardy space $H^{p}(\mathbb{B})$ is defined as

$$
H^{p}(\mathbb{B})=\left\{f: \mathbb{B} \rightarrow \mathbb{H} \mid f \text { is regular and }\|f\|_{p}<\infty\right\} .
$$

Obviously, $H^{p}(\mathbb{B})$ is a real vector space. Moreover, $H^{p}(\mathbb{B})$ is not only a left $\mathbb{H}$-module, but also a right $\mathbb{H}$-module with respect to + and $*$. In analogy with the complex case, the space $H^{2}(\mathbb{B})$ is a Hilbert $\mathbb{H}$-module (see 9 ). 
Proposition 2.18. Let $f \in H^{2}(\mathbb{B})$ and let $f(q)=\sum_{n=0}^{\infty} q^{n} a_{n}$ be its power series expansion. Then the 2-norm of $f$,

$$
\|f\|_{2}=\sup _{I \in \mathbb{S}} \lim _{r \rightarrow 1^{-}}\left(\frac{1}{2 \pi} \int_{-\pi}^{\pi}\left|f\left(r e^{I \theta}\right)\right|^{2} d \theta\right)^{\frac{1}{2}}
$$

coincides with

$$
\left(\sum_{n=0}^{\infty}\left|a_{n}\right|^{2}\right)^{\frac{1}{2}} .
$$

Moreover, for any $r \in[0,1)$ the integral

$$
\frac{1}{2 \pi} \int_{-\pi}^{\pi}\left|f\left(r e^{I \theta}\right)\right|^{2} d \theta=\sum_{n=0}^{\infty}\left|a_{n}\right|^{2} r^{2 n}
$$

does not depend on $I \in \mathbb{S}$.

\section{VlacCi's REGULAR COMPOSITION}

One point in the setting of quaternions is that the regularity does not keep under the usual composition. Indeed, the only case in which the regularity of $f \circ \varphi$ is maintained for any regular function $f$ is when the regular function $\varphi$ is slice preserving. If instead $\varphi$ has no constraints, then $f$ has to be an affine function of the form $f(q)=a+q b$ for some $a, b \in \mathbb{H}$.

Vlacci [26] introduces a kind of regular composition for regular functions, which preserves regularity locally.

Let $f: B(0, R) \rightarrow \mathbb{H}$ be regular with Taylor expansion

$$
f(q)=\sum_{n=0}^{\infty} q^{n} a_{n}
$$

and let $\varphi: B(0, r) \rightarrow B(0, R)$ be another regular function with $0<r \leq \infty$ and

$$
\varphi(q)=\sum_{n=0}^{\infty} q^{n} b_{n}
$$

Starting from the Taylor expansion

$$
f^{\circ} \varphi(q)=\sum_{n=0}^{\infty} q^{n} \frac{\left(f^{\circ} \varphi\right)^{(n)}(0)}{n !}
$$

and inspired by the classical Fà di Bruno formula in the commutative setting, Vlacci introduces the following regular composition of $f$ with $\varphi$.

Definition 3.1. The Vlacci's regular composition of $f$ with $\varphi$ is formally defined by

$$
f^{\circ} \varphi(q)=\sum_{n=0}^{\infty} q^{n} c_{n},
$$

whose coefficients are given by

$$
c_{0}=f\left(b_{0}\right)
$$


and

$$
c_{n}=\frac{1}{n !} \sum_{d=1}^{n} B_{n, d}\left(b_{1}, 2 ! b_{2}, \cdots, n ! b_{n}\right) f^{(d)}\left(b_{0}\right), \quad n \geq 1 .
$$

Here $B_{n, d}(d=1,2, \cdots, n)$ are the homogeneous parts of the corresponding noncommutative Bell polynomial $B_{n}$, which coincides with the classical Bell polynomial when restricted to $\mathbb{C}^{n}$.

Denote

$$
C\left(n, n_{2}, \ldots, n_{d}\right)=\left(\begin{array}{c}
n-1 \\
n_{2}
\end{array}\right)\left(\begin{array}{c}
n_{2}-1 \\
n_{3}
\end{array}\right) \cdots\left(\begin{array}{c}
n_{d-1}-1 \\
n_{d}
\end{array}\right)
$$

The explicit expression of $B_{n, d}$ is given by

$$
B_{n, d}\left(q_{1}, \cdots, q_{n}\right)=\sum_{n>n_{2}>\cdots>n_{d} \geq 1} C\left(n, n_{2}, \ldots, n_{d}\right) q_{n_{d}} q_{n_{d-1}-n_{d}} \cdots q_{n_{2}-n_{3}} q_{n-n_{2}}
$$

for $n \geq d \geq 2$ and

$$
B_{n-1,0}=1 \quad \text { and } \quad B_{n, 1}\left(q_{1}, q_{2}, \ldots, q_{n}\right)=q_{n}, \quad n \geq 1 .
$$

These can be verified by applying the recursion equation

$$
B_{n+1}=\sum_{k=0}^{n}\left(\begin{array}{l}
n \\
k
\end{array}\right) B_{n-k} q_{k+1}
$$

for $n=0,1,2, \cdots$ together with the initial condition

$$
B_{0}=1 \text {. }
$$

For more generally noncommutative setting, not only limited to quaternions, we refer to [24, Theorem 1] and the references therein for more details.

With the Vlacci approach, one can introduce some new regular compositions. For example, one can modify Vlacci's regular composition by changing the order of the factors in the summands of (3.2). We thus can introduce new regular composition of $f$ with $\varphi$, denoted by $f_{\circ} \varphi$, via

$$
f_{\circ} \varphi(q)=\sum_{n=0}^{\infty} q^{n} d_{n}
$$

whose coefficients are given by

$$
d_{0}=f\left(b_{0}\right), \quad d_{n}=\frac{1}{n !} \sum_{d=1}^{n} f^{(d)}\left(b_{0}\right) B_{n, d}\left(b_{1}, 2 ! b_{2}, \cdots, n ! b_{n}\right), \quad n \geq 1 .
$$

At first sight, it seems that the definition of $f^{\circ} \varphi$ coincides with that of $f_{\circ} \varphi$. Unfortunately, the answer is negative in general, as the following example shown.

Example 3.2. Let $f(q)=q$ and $\varphi(q)=q^{2} I$ with $I, J \in \mathbb{S}$ and $I \perp J$, then

$$
f^{\circ} \varphi(q)=q^{2} I J, \quad \text { while } \quad f_{\circ} \varphi(q)=-q^{2} I J .
$$

Some new regular compositions can be further introduced. Motivated by (4.2) below one can introduce regular compositions $f \bullet \varphi$ and $f^{\bullet} \varphi$ via identities

$$
\left(f^{\bullet} \varphi\right)^{c}=\left(f^{c}\right)_{\circ}\left(\varphi^{c}\right), \quad(f \bullet \varphi)^{c}=\left(f^{c}\right)^{\circ}\left(\varphi^{c}\right) .
$$

Here we use the fact that regular conjugations are involution operators. 
There are strict restraints for the existence of the above slice regular compositions.

For any regular function $f(q)=\sum_{n=0}^{\infty} q^{n} a_{n}$ on $B(0, R)$, denote

$$
f_{a b s}(q)=\sum_{n=0}^{\infty} q^{n}\left|a_{n}\right| .
$$

Vlacci [26] provides a sufficient condition for which the regular compositions exist.

Theorem 3.3. If the composition $f_{a b s} \circ \varphi_{a b s}$ exists on $B(0, R)$, then $f^{\circ} \varphi$ is regular on $B(0, R)$.

However, the Bohr-type phenomenon [22, 14] shows that

$$
\varphi_{\text {abs }}: \frac{1}{3} \mathbb{B} \longrightarrow \mathbb{B}
$$

for any regular function $\varphi: \mathbb{B} \longrightarrow \mathbb{B}$. Since the range of the function $\varphi_{a b s}$ plays a role in the above composition, we thus observed that there exists obstacle to define

$$
\varphi_{a}^{\circ} \varphi_{a}
$$

Here $\varphi_{a}$ is the regular Möbius transformation of $\mathbb{B}$ (see (7.4)).

\section{Slice Regular compositions}

Vlacci's slice regular composition is defined only locally, we need to introduce a globally defined slice regular composition in order to achieve the theory of regular composition.

Definition 4.1. Let $\Omega$ be a symmetric slice domain in $\mathbb{H}$ and $0<R \leq \infty$. For any two regular functions $\varphi: \Omega \rightarrow B(0, R)$ and $f: B(0, R) \rightarrow \mathbb{H}$ with power series expansion

$$
f(q)=\sum_{n=0}^{\infty} q^{n} a_{n}
$$

a regular composition of $f$ with $\varphi$ can be defined by

$$
f^{\odot} \varphi(q)=\sum_{n=0}^{\infty} \varphi^{* n}(q) a_{n} .
$$

Alternatively, one can also define regular composition of $f$ with $\varphi$ as

$$
f_{\odot} \varphi(q)=\sum_{n=0}^{\infty} a_{n} * \varphi^{* n}(q) .
$$

These two regular compositions are different and conjugate in some sense, see Theorem 4.12. This fact reflects the diversity of the noncommutative setting as we mentioned in the introduction.

Some remarks are in order.

Remark 4.2. The two regular compositions of $f$ with $\varphi$ are both well-defined. Indeed, for any fixed $q_{0}=x+y I \in \Omega$, let $M$ denote the maximum modulus of $\varphi$ on the 2-sphere $\left[q_{0}\right]=\{x+y J: J \in \mathbb{S}\}$, i.e.

$$
M=\max _{q \in\left[q_{0}\right]}|\varphi(q)|<R,
$$


since $\varphi(\Omega) \subseteq B(0, R)$. From Proposition 2.9 it is easy to prove by induction that

$$
\left|\varphi^{* n}(q)\right| \leq M^{n}
$$

for any $q \in\left[q_{0}\right]$ and any $n \in \mathbb{N}$. Again Proposition 2.9 implies that for all $q \in\left[q_{0}\right]$,

$$
\sum_{n=0}^{\infty}\left|\varphi^{* n}(q) a_{n}\right| \leq \sum_{n=0}^{\infty}\left|a_{n}\right| M^{n}<\infty
$$

and

$$
\sum_{n=0}^{\infty}\left|a_{n} * \varphi^{* n}(q)\right| \leq \sum_{n=0}^{\infty}\left|a_{n}\right| M^{n}<\infty
$$

owing to the absolute and uniform convergence of the power series $\sum_{n=0}^{\infty} q^{n} a_{n}$ on the closed ball $\overline{B(0, M)} \subset B(0, R)$.

Remark 4.3. If $\varphi$ is a slice preserving function, i.e. $\varphi\left(\Omega_{I}\right) \subseteq \mathbb{C}_{I}$ for any $I \in \mathbb{S}$, then the two kinds of regular compositions $f^{\odot} \varphi$ and $f_{\odot} \varphi$ both coincide with the usual composition $f \circ \varphi$.

Remark 4.4. At first sight, it seems that the definitions of $f^{\circ} \varphi$ and $f_{\circ} \varphi$ coincide with those of $f^{\odot} \varphi$ and $f_{\odot} \varphi$ respectively. Unfortunately, the answer is negative in general, as shown by the following example.

Example 4.5. Let $f(q)=q^{2}$ and $\varphi(q)=q^{2} I+q J$ with $I, J \in \mathbb{S}$ and $I \perp J$. Then a straightforward calculation gives

$$
f^{\odot} \varphi(q)=f_{\odot} \varphi(q)=q^{2}(q I+J)^{* 2}=-q^{4}-q^{2},
$$

and

$$
f^{\circ} \varphi(q)=f_{\circ} \varphi(q)=-q^{4}-\frac{2}{3} q^{3} I J-q^{2} .
$$

Remark 4.6. As mentioned in the end of the preceding section, for a general regular function $f$, the radii of convergence of power series in (3.1) are unknown and it is quite possible that the definition domains of $f^{\circ} \varphi$ may be quite small than that of $\varphi$.

The advantages of new regular composition over Vlacci's is that $f_{\odot} \varphi$ has the same definition domain with that of $\varphi$, under the right hypotheses about the domain and range of $f$ and $\varphi$. Moreover, the definition domain of $\varphi$ is only required to be a symmetric slice domain other than a ball.

Remark 4.7. The two regular compositions $f^{\odot} \varphi$ and $f_{\odot} \varphi$ are different and both not, in general, associative, as the following example shown.

Example 4.8. Let $f(q)=q^{2}, g(q)=1+q I$ and $\varphi(q)=q J$ with $I, J \in \mathbb{S}$ and $I \perp J$. Then a straightforward calculation gives

$$
\left(f^{\odot} g\right)^{\odot} \varphi(q)=q^{2}+2 q J I+1 \quad \text { and } \quad f^{\odot}\left(g^{\odot} \varphi\right)(q)=-q^{2}+2 q J I+1,
$$

while

$$
\left(f_{\odot} g\right) \odot \varphi(q)=q^{2}+2 q I J+1 \quad \text { and } \quad f_{\odot}\left(g_{\odot} \varphi\right)(q)=-q^{2}+2 q I J+1,
$$

which tell us that

$$
\left(f^{\odot} g\right)^{\odot} \varphi \neq f^{\odot}\left(g^{\odot} \varphi\right) \quad \text { and } \quad\left(f_{\odot} g\right)_{\odot} \varphi \neq f_{\odot}\left(g_{\odot} \varphi\right) .
$$


Moreover,

$$
g^{\odot} \varphi(q)=1+q J I, \quad \text { while } \quad g_{\odot} \varphi(q)=1+q I J,
$$

which shows that

$$
g^{\odot} \varphi \neq g_{\odot} \varphi .
$$

Remark 4.9. The quaternionic counterparts of the identity

$$
(f g) \circ \varphi=(f \circ \varphi)(g \circ \varphi)
$$

do not always hold. Namely, in general,

$$
(f * g)^{\odot} \varphi \neq\left(f^{\odot} \varphi\right) *\left(g^{\odot} \varphi\right), \quad(f * g)_{\odot} \varphi \neq(f \odot \varphi) *\left(g_{\odot} \varphi\right),
$$

as shown by the following example.

Example 4.10. Let $f(q)=q^{2} I, g(q)=1+q J, \varphi(q)=q I J$ and $\psi(q)=q+I$ with $I, J \in \mathbb{S}$ and $I \perp J$. Then a straightforward calculation gives

$(f * g)^{\odot} \varphi(q)=q^{3}-q^{2} I, \quad(f * g) \odot \psi(q)=q^{3} I J+q^{2}(I+3 J)-q(3 I J+2)-I-J$, while

$\left(f^{\odot} \varphi\right) *\left(g^{\odot} \varphi\right)(q)=-q^{3}-q^{2} I, \quad\left(f_{\odot} \psi\right) *\left(g_{\odot} \psi\right)(q)=q^{3} I J+q^{2}(I-J)+q(I J-2)-I-J$, which shows that

$$
(f * g)^{\odot} \varphi \neq\left(f^{\odot} \varphi\right) *\left(g^{\odot} \varphi\right), \quad(f * g) \odot \psi \neq\left(f_{\odot} \psi\right) *(g \odot \psi) .
$$

However, the following property holds.

Proposition 4.11. Let $f, g$ be two regular functions on $B(0, R)$ and Let $\varphi: \Omega \rightarrow$ $B(0, R)$ be regular on a symmetric slice domain. Then

$$
(f * g)^{\odot} \varphi=\left(f^{\odot} \varphi\right) *\left(g^{\odot}\left(\left(f^{\odot} \varphi\right)^{-*} * \varphi *\left(f^{\odot} \varphi\right)\right)\right) \quad \text { on } \Omega \backslash \mathcal{Z}_{(f \odot \varphi)},
$$

and

$$
(f * g)_{\odot} \varphi=\left(f_{\odot}\left(\left(g_{\odot} \varphi\right) * \varphi *\left(g_{\odot} \varphi\right)^{-*}\right)\right) *\left(g_{\odot} \varphi\right) \quad \text { on } \Omega \backslash \mathcal{Z}_{\left(g_{\odot} \varphi\right)^{s}} .
$$

Proof. Let $f, g: \mathbb{B} \rightarrow \mathbb{H}$ be as described with Taylor expansion of the form

$$
f(q)=\sum_{n=0}^{\infty} q^{n} a_{n}, \quad g(q)=\sum_{n=0}^{\infty} q^{n} b_{n}
$$

respectively. From the very definition,

$$
\begin{aligned}
(f * g)^{\odot} \varphi & =\sum_{n=0}^{\infty} \varphi^{* n}\left(\sum_{k=0}^{n} a_{n-k} b_{k}\right)=\sum_{k=0}^{\infty} \varphi^{* k} *\left(\sum_{n=0}^{\infty} \varphi^{* n} a_{n}\right) b_{k} \\
& =\sum_{k=0}^{\infty} \varphi^{* k} *\left(f^{\odot} \varphi\right) b_{k}=\left(f^{\odot} \varphi\right) * \sum_{k=0}^{\infty}\left(\left(f^{\odot} \varphi\right)^{-*} * \varphi *\left(f^{\odot} \varphi\right)\right)^{* k} b_{k} \\
& =\left(f^{\odot} \varphi\right) *\left(g^{\odot}\left(\left(f^{\odot} \varphi\right)^{-*} * \varphi *\left(f^{\odot} \varphi\right)\right)\right)
\end{aligned}
$$

The other one can also be proved similarly and an alternative way can be achieved immediately by applying the following proposition.

In contrast to the local existence of Vlacci's composition as shown in Theorem 3.3. our slice regular compositions exists globally. 
Proposition 4.12. Let $\Omega$ be a symmetric slice domain in $\mathbb{H}$ and $0<R \leq \infty$. For any two regular functions $f: B(0, R) \rightarrow \mathbb{H}$ and $\varphi: \Omega \rightarrow B(0, R)$, we have $f^{\odot} \varphi$ and $f_{\odot} \varphi$ exist and are regular on $\Omega$ satisfying

$$
\left(f^{\odot} \varphi\right)^{c}=f_{\odot}^{c} \varphi^{c}, \quad\left(f_{\odot} \varphi\right)^{c}=f^{c} \odot \varphi^{c} .
$$

Proof. First, we prove that both $f^{c} \odot \varphi^{c}$ and $f^{c}{ }_{\odot} \varphi^{c}$ are well-defined on $\Omega$. According to Remark 4.2, it suffices to prove that $\varphi^{c}(\Omega) \subseteq B(0, R)$. Indeed, suppose $\varphi^{c}(p)=$ $a \in \mathbb{H} \backslash B(0, R)$ for some $p=x+I y \in \Omega$. Then $p$ is a zero of the regular function $\varphi^{c}-a$. By Proposition 2.12, there exists $\widetilde{p} \in x+y \mathbb{S} \subseteq \Omega$ such that $\left(\varphi^{c}-a\right)^{c}=\varphi-\bar{a}$ vanishes at $\widetilde{p}$. Hence, $\varphi(\Omega)$ includes $\bar{a} \in \mathbb{H} \backslash B(0, R)$, which is a contradiction with the hypothesis $\varphi(\Omega) \subseteq B(0, R)$.

Let $\sum_{n=0}^{\infty} q^{n} a_{n}$ be power series expansion of $f$, i.e. $f(q)=\sum_{n=0}^{\infty} q^{n} a_{n}$. According to the definition of regular composition,

$$
\left(f^{\odot} \varphi\right)^{c}=\sum_{n=0}^{\infty}\left(\varphi^{* n} a_{n}\right)^{c}=\sum_{n=0}^{\infty} \bar{a}_{n} *\left(\varphi^{c}\right)^{* n}=f_{\odot}^{c} \varphi^{c},
$$

and

$$
\left(f_{\odot} \varphi\right)^{c}=\sum_{n=0}^{\infty}\left(a_{n} * \varphi^{* n}\right)^{c}=\sum_{n=0}^{\infty}\left(\varphi^{c}\right)^{* n} \bar{a}_{n}=f^{c} \odot \varphi^{c}
$$

as desired.

The approach in the proof of Theorem 4.12 also immediately works to extend Proposition 2.13 from the unit ball to symmetric slice domains.

Proposition 4.13. Let $f: \Omega_{1} \rightarrow \Omega_{2}$ be a regular function with $\Omega_{i} \subseteq \mathbb{H}(i=1,2)$ two symmetric slice domains. Then the regular conjugate function $f^{c}: \Omega_{1} \rightarrow \mathbb{H}$ has the following properties.

(i) $f^{c}$ is regular satisfying $f^{c}\left(\Omega_{1}\right) \subset \Omega_{2}$.

(ii) $f^{c}$ is bijective if and only if $f$ is.

(iii) For any $x, y \in \mathbb{R}$ such that $x+y \mathbb{S} \subset \Omega_{1}$,

$$
\sup _{I \in \mathbb{S}}|f(x+y I)|=\sup _{I \in \mathbb{S}}\left|f^{c}(x+y I)\right|, \quad \inf _{I \in \mathbb{S}}|f(x+y I)|=\inf _{I \in \mathbb{S}}\left|f^{c}(x+y I)\right|
$$

(iv) There holds the identity

$$
\sup _{q \in \Omega_{1}}|f(q)|=\sup _{q \in \Omega_{1}}\left|f^{c}(q)\right|, \quad \inf _{q \in \Omega_{1}}|f(q)|=\inf _{q \in \Omega_{1}}\left|f^{c}(q)\right| .
$$

\section{The Denjoy-Wolff type theorem}

The dynamical behaviors of the iterates of regular self-mappings $f$ of $\mathbb{B}$ is considered in this section, under the extra assumption that $f$ preserves at least one slice. The limit of the iterates turns out to be the regular Möbius transformation of the unit ball.

In view of Theorem 5.4 below, it is useful to recall the following definition given in [19] and there Gentili and Vlacci provide a complete description of the fixed-point set for regular Möbius transformations of a quaternionic variable.

Definition 5.1. A regular Möbius transformation of $\mathbb{B}$ with only one fixed point in $\mathbb{B}$ is called elliptic.

A regular Möbius transformation of $\mathbb{B}$ without fixed points in $\mathbb{B}$ is called: 
- parabolic if it has only one fixed point on the boundary of $\mathbb{B}$;

- hyperbolic if it has at least two fixed points on the boundary of $\mathbb{B}$.

In particular, a hyperbolic regular Möbius transformation of $\mathbb{B}$ with a sphere of fixed points on the boundary of $\mathbb{B}$ is called spherical-hyperbolic.

It is crucial to show the existence of iterates of regular self-mappings with respect to our slice regular compositions.

Theorem 5.2. Let $f$ be a regular self-mapping of $\mathbb{B}$ such that $f\left(\mathbb{B}_{I}\right) \subseteq \mathbb{B}_{I}$ for some $I \in \mathbb{S}$, then $f^{\odot n}$ is well defined as a regular self-mapping of $\mathbb{B}$ and

$$
f^{\odot n}=\operatorname{ext}\left(f_{I}\right)^{n}
$$

for any $n \in \mathbb{N}$.

Proof. Let $f: \mathbb{B} \rightarrow \mathbb{B}$ be given as described.

First, we prove that $\operatorname{ext}\left(f_{I}\right)^{n}$ is well defined as a regular self-mapping of $\mathbb{B}$ for any $n \in \mathbb{N}$. Indeed, since the restriction $f_{I}$ of $f$ to $\mathbb{B}_{I}$ is a holomorphic self-mapping of the open unit disc $\mathbb{B}_{I} \subset \mathbb{C}_{I}$, then we can consider the iterates $\left(f_{I}\right)^{n}$ of $f_{I}$, where $\left(f_{I}\right)^{1}=f_{I}$ and

$$
\left(f_{I}\right)^{n+1}=f_{I} \circ\left(f_{I}\right)^{n}, \quad n=1,2, \cdots .
$$

Consequently, for each $n \in \mathbb{N},\left(f_{I}\right)^{n}$ is a holomorphic self-mapping of $\mathbb{B}_{I}$, which naturally induces a regular self-mapping of $\mathbb{B}$, say $\operatorname{ext}\left(f_{I}\right)^{n}$, by using regular extension. The assertion that $\operatorname{ext}\left(f_{I}\right)^{n}(\mathbb{B}) \subseteq \mathbb{B}$ follows from a convex combination identity in Lemma 3.3 of [21].

Next, we prove that $f^{\odot n}$ is well defined and

$$
f^{\odot n}=\operatorname{ext}\left(f_{I}\right)^{n}
$$

for any $n \in \mathbb{N}$. The reasons are as following. We can formally define in any order the $n$-th regular composition of $f$, on some small neighborhood $\mathcal{O}_{n} \subset \mathbb{B}$ of 0 , via (4.1), and then it suffices to prove that the $n$th regular composition in any order can regularly extend to $\mathbb{B}$ and coincides with $\operatorname{ext}\left(f_{I}\right)^{n}$ there.

For simplicity, we only consider the $n$th regular composition

$$
\underbrace{f^{\odot}\left(\cdots\left(f^{\odot}\left(f^{\odot} f\right)\right)\right)}_{n \text { copies }}
$$

and from the very definition its restriction

$$
\left(f^{\odot}\left(\cdots\left(f^{\odot}\left(f^{\odot} f\right)\right)\right)\right)_{I}
$$

to $\left(\mathcal{O}_{n}\right)_{I}$ coincides with $\left(f_{I}\right)^{n}$ there, but $\left(f_{I}\right)^{n}$ and its regular extension $\operatorname{ext}\left(f_{I}\right)^{n}$ are well defined on $\mathbb{B}_{I}$ and $\mathbb{B}$, respectively. Therefore, we can regularly extend $f \odot(\cdots(f \odot(f \odot f)))$ to the whole ball $\mathbb{B}$ and

$$
f^{\odot}\left(\cdots\left(f^{\odot}\left(f^{\odot} f\right)\right)\right)=\operatorname{ext}\left(f_{I}\right)^{n} .
$$

Similarly, we can use the same arguments as before to prove that the $n$th regular composition in any other order is well defined on $\mathbb{B}$ and coincides with $\operatorname{ext}\left(f_{I}\right)^{n}$ as well. Thus we can denote by $f^{\odot n}$ the well defined $n$th regular composition for any $n \in \mathbb{N}$.

Now we consider the slice iterates. 
Proposition 5.3. Let $f$ be a regular self-mapping such that $f\left(\mathbb{B}_{I}\right) \subseteq \mathbb{B}_{I}$ for some $I \in \mathbb{S}$ and suppose $\left\{f^{\odot n}\right\}$ has a subsequence which converges to a nonconstant function. Then $f$ is a regular Möbius transformation of $\mathbb{B}$.

Proof. Let $f: \mathbb{B} \rightarrow \mathbb{B}$ be as described. Then the sequence $\left\{\left(f^{\odot n}\right)_{I}\right\}=\left\{\left(f_{I}\right)^{n}\right\}$ satisfies the assumption given in Lemma 2.50 of $\left[8\right.$, thus $f_{I} \in \operatorname{Aut}\left(\mathbb{B}_{I}\right)$ and its regular extension $f=\operatorname{ext}\left(f_{I}\right)$ is a regular Möbius transformation of $\mathbb{B}$.

Moreover, we have the following Denjoy-Wolff type theorem.

Theorem 5.4. (Denjoy-Wolff Type Theorem) Let $I d \neq f \in A u t(\mathbb{B})$ be not elliptic automorphism in which $f\left(\mathbb{B}_{I}\right) \subseteq \mathbb{B}_{I}$ for some $I \in \mathbb{S}$, then the sequence of $\left\{f^{\odot n}\right\}$ uniformly converges on every compact subset of $\mathbb{B}$ to a boundary fixed point of $f$.

Proof. For any fixed compact subset $C \subset \mathbb{B}$, there exists $r \in(0,1)$ such that $C \subseteq \overline{B(0, r)} \subset \mathbb{B}$. By assumption, the sequence $\left\{\left(f^{\odot n}\right)_{I}\right\}=\left\{\left(f_{I}\right)^{n}\right\}$ satisfies the assumption given in Theorem 2.51 of [8], thus $\left\{\left(f_{I}\right)^{n}\right\}$ uniformly converges on compact set $\overline{(B(0, r))_{I}} \subset \mathbb{B}_{I}$ to a boundary fixed point of $f$. By a convex combination identity from Lemma 3.3 of [21,

$$
\max _{q \in \overline{B(0, r)}}|f(q)-a|=\max _{z \in \overline{(B(0, r))_{I}}}|f(z)-a|,
$$

which gives the uniform convergence of $\left\{f^{\odot n}\right\}$ on $C$, since $C \subseteq \overline{B(0, r)}$.

Alternatively, the uniform convergence of $\left\{f^{\odot n}\right\}$ on $C \subset \mathbb{B}$ can follow from the representation formula.

\section{The LitTlewood Subordination PRINCiPle}

In this section we establish the Littlewood subordination principle for regular functions.

Theorem 6.1. (Littlewood Subordination Principle) Let $\varphi: \mathbb{B} \rightarrow \mathbb{B}$ be a regular function and $\varphi(0)=0$, then

$$
\left\|f^{\odot} \varphi\right\|_{2} \leq\|f\|_{2}, \quad\left\|f_{\odot} \varphi\right\|_{2} \leq\|f\|_{2}
$$

for any $f \in H^{2}(\mathbb{B})$.

To prove this result, we need some basic lemmas.

Lemma 6.2. Let $f \in H^{2}(\mathbb{B})$, then for all $q \in \mathbb{B}$,

$$
|f(q)| \leq\left(\frac{1}{1-|q|^{2}}\right)^{\frac{1}{2}}\|f\|_{2} .
$$

Proof. Consider the power series expansion of $f$

$$
f(q)=\sum_{n=0}^{\infty} q^{n} \hat{f}(n) .
$$

By the Cauchy-Schwarz inequality, we have

$$
|f(q)| \leq \sum_{n=0}^{\infty}|q|^{n}|\hat{f}(n)| \leq\left(\sum_{n=0}^{\infty}|\hat{f}(n)|^{2}\right)^{\frac{1}{2}}\left(\sum_{n=0}^{\infty}|q|^{2 n}\right)^{\frac{1}{2}}=\left(\frac{1}{1-|q|^{2}}\right)^{\frac{1}{2}}\|f\|_{2}
$$


for all $q \in \mathbb{B}$.

Lemma 6.3. Let $\left\{f_{n}\right\}_{n \in \mathbb{N}}$ be a convergent sequence in $H^{2}(\mathbb{B})$. Then $\left\{f_{n}\right\}_{n \in \mathbb{N}}$ converges uniformly on every compact subset of $\mathbb{B}$.

Proof. Suppose that $f_{n} \longrightarrow f \in H^{2}(\mathbb{B})$, i.e. $\left\|f_{n}-f\right\|_{2} \longrightarrow 0$ as $n \longrightarrow \infty$, then for any compact set $C \subset \mathbb{B}$, there exists $r \in(0,1)$ such that $C \subseteq \overline{B(0, r)} \subset \mathbb{B}$, by Theorem 6.2 and the Maximum Modulus Principle 2.15 we have

$$
\sup _{q \in C}\left|f_{n}(q)-f(q)\right| \leq \sup _{|q| \leq r}\left|f_{n}(q)-f(q)\right| \leq\left(\frac{1}{1-r^{2}}\right)^{\frac{1}{2}}\|f\|_{2} \longrightarrow 0 \quad \text { as } n \longrightarrow \infty,
$$

which implies that $\left\{f_{n}\right\}_{n \in \mathbb{N}}$ converges uniformly to $f$ on $C$.

Lemma 6.4. Let $f \in H^{2}(\mathbb{B})$ and $\varphi \in H^{\infty}(\mathbb{B})$, then $f * \varphi, \varphi * f \in H^{2}(\mathbb{B})$. Moreover,

$$
\begin{aligned}
& \|f * \varphi\|_{2} \leq\|\varphi\|_{\infty}\|f\|_{2}, \\
& \|\varphi * f\|_{2} \leq\|\varphi\|_{\infty}\|f\|_{2} .
\end{aligned}
$$

Proof. By Proposition 2.9, we have for every $q \in \mathbb{B}$,

$$
|f * \varphi(q)| \leq|f(q)|\|\varphi\|_{\infty}
$$

and hence for any $0<p<\infty$,

$$
\begin{aligned}
\|f * \varphi\|_{p} & =\sup _{I \in \mathbb{S}} \lim _{r \rightarrow 1^{-}}\left(\frac{1}{2 \pi} \int_{-\pi}^{\pi}\left|f * \varphi\left(r e^{I \theta}\right)\right|^{p} d \theta\right)^{\frac{1}{p}} \\
& \leq\|\varphi\|_{\infty} \sup _{I \in \mathbb{S}} \lim _{r \rightarrow 1^{-}}\left(\frac{1}{2 \pi} \int_{-\pi}^{\pi}\left|f\left(r e^{I \theta}\right)\right|^{p} d \theta\right)^{\frac{1}{p}} \\
& =\|\varphi\|_{\infty}\|f\|_{p} .
\end{aligned}
$$

In particular,

$$
\|f * \varphi\|_{2} \leq\|\varphi\|_{\infty}\|f\|_{2} .
$$

As for $f * \varphi$, according to Proposition 2.18 and Proposition 4.13 that

$$
\left\|f^{c}\right\|_{2}=\|f\|_{2} \quad \text { and } \quad\left\|\varphi^{c}\right\|_{\infty}=\|\varphi\|_{\infty}
$$

Together with (6.1) and the fact that $(\varphi * f)^{c}=f^{c} * \varphi^{c}$, these yield

$$
\|\varphi * f\|_{2}=\left\|(\varphi * f)^{c}\right\|_{2}=\left\|f^{c} * \varphi^{c}\right\|_{2} \leq\left\|\varphi^{c}\right\|_{\infty}\left\|f^{c}\right\|_{2}=\|\varphi\|_{\infty}\|f\|_{2},
$$

which completes the proof.

Incidentally, the approach to the proof of the preceding lemma also immediately give a very simple proof of Theorem 5.17 in [1, which is of independent interest, and is initially proved for the left multiplier in the special case that $p=2$ by using an approximation argument and Runge's theorem.

Proposition 6.5. Let $\varphi_{a}$ is a regular Möbius transformation of $\mathbb{B}$. then the multiplier operators

$$
\mathcal{M}_{\varphi_{a}}^{l}: f \mapsto \varphi_{a} * f
$$

and

$$
\mathcal{M}_{\varphi_{a}}^{r}: f \mapsto f * \varphi_{a}
$$

are isometries on $H^{2}(\mathbb{B})$ and $H^{\infty}(\mathbb{B})$, respectively. Furthermore, $\mathcal{M}_{\varphi_{a}}^{r}$ is an isometry on $H^{p}(\mathbb{B})$ for $p \neq 2$. 
Proof. From [25, we know every regular Möbius transformation $\varphi_{a}$ of $\mathbb{B}$ is of the form

$$
\varphi_{a}(q)=(1-q \bar{a})^{-*} *(a-q) u=: M_{a} u,
$$

where $u \in \partial \mathbb{B}$. For any $f \in H^{2}(\mathbb{B})$, it follows from Proposition 2.18 that

$\left\|\mathcal{M}_{\varphi_{a}}^{l} f\right\|_{2}=\left\|\left(\mathcal{M}_{\varphi_{a}}^{l} f\right)_{I}\right\|_{2}=\left\|\left(M_{\varphi_{a}}\right)_{I}(u * f)_{I}\right\|_{2}=\left\|(u * f)_{I}\right\|_{2}=\|u * f\|_{2}=\|f\|_{2}$

and

$$
\left\|\mathcal{M}_{\varphi_{a}}^{r} f\right\|_{2}=\left\|\left(\mathcal{M}_{\varphi_{a}}^{r} f\right)^{c}\right\|_{2}=\left\|\left(\varphi_{a}\right)^{c} * f^{c}\right\|_{2}=\left\|f^{c}\right\|_{2}=\|f\|_{2} .
$$

The penultimate equation follows from the fact that $\left(\varphi_{a}\right)^{c}$ is also a regular Möbius transformation of $\mathbb{B}$ and what we just proved on the isometry property of left multiplier operators.

The fact that both $\mathcal{M}_{a}^{l}$ and $\mathcal{M}_{a}^{r}$ are isometries from $H^{\infty}(\mathbb{B})$ into itself follows immediately from Proposition 2.9 and (iv) in Proposition 4.13.

It remains to prove that $\mathcal{M}_{\varphi_{a}}^{r}$ is an isometry on $H^{p}(\mathbb{B})$ for $p \neq 2$. By the proof of Lemma 6.4, it suffices to prove that

$$
\left\|\mathcal{M}_{\varphi_{a}}^{r} f\right\|_{p} \geq\|f\|_{p}
$$

for any $f \in H^{p}(\mathbb{B})$ with $\|f\|_{p}>0$. For any fixed $\varepsilon \in\left(0,\|f\|_{p}\right)$, from the very definition it follows that there are some $I_{0} \in \mathbb{S}$ and some $r_{0} \in(0,1)$ such that

$$
\left(\frac{1}{2 \pi} \int_{-\pi}^{\pi}\left|f\left(r_{0} e^{I_{0} \theta}\right)\right|^{p} d \theta\right)^{\frac{1}{p}} \geq\|f\|_{p}-\varepsilon
$$

which together with Proposition 2.9 yields that

$$
\left\|\mathcal{M}_{\varphi_{a}}^{r} f\right\|_{p} \geq\left(\frac{1}{2 \pi} \int_{-\pi}^{\pi}\left|f * \varphi_{a}\left(r e^{I_{0} \theta}\right)\right|^{p} d \theta\right)^{\frac{1}{p}} \geq\left(\|f\|_{p}-\varepsilon\right) \min _{|q|=r}\left|\varphi_{a}(q)\right|
$$

for any $r \in\left(r_{0}, 1\right)$. Therefore, letting $r \longrightarrow 1$ yields

$$
\left\|\mathcal{M}_{\varphi_{a}}^{r} f\right\|_{p} \geq\|f\|_{p}-\varepsilon
$$

which completes the proof.

We are now in a position to prove the main theorem of this section.

Proof of Theorem 6.1. We define a translation operator $T: H^{2}(\mathbb{B}) \rightarrow H^{2}(\mathbb{B})$ via

$$
(T f)(q)=\sum_{n=0}^{\infty} q^{n} \hat{f}(n+1)
$$

for any $f \in H^{2}(\mathbb{B})$ with the Taylor expansion

$$
f(q)=\sum_{n=0}^{\infty} q^{n} \hat{f}(n) \in H^{2}(\mathbb{B}) .
$$

Due to Proposition 2.18,

$$
\|T f\|_{2}^{2}=\sum_{n=0}^{\infty}|\hat{f}(n+1)|^{2}=\sum_{n=1}^{\infty}|\hat{f}(n)|^{2} \leq\|f\|_{2}^{2}
$$

and hence $T f \in H^{2}(\mathbb{B})$. Moreover, by definition,

$$
f(q)=f(0)+q(T f)(q), \quad \forall q \in \mathbb{B},
$$


and

$$
\left(T^{n} f\right)(0)=\hat{f}(n), \quad \forall n \in \mathbb{N} .
$$

for any $f \in H^{2}(\mathbb{B})$.

We suppose first that $f$ is a regular polynomial. In this case, Proposition 2.9 shows that $f^{\odot} \varphi$ is bounded on $\mathbb{B}$ and so is in $H^{2}(\mathbb{B})$. Now we estimate its norm $\left\|f^{\odot} \varphi\right\|_{2}$. By (6.2) we have

$$
f^{\odot} \varphi(q)=f(0)+\varphi *\left((T f)^{\odot} \varphi\right)(q), \quad \forall q \in \mathbb{B} .
$$

By assumption $\varphi(0)=0$, which implies that the second term on the right-hand side of the identity above takes value 0 at $q=0$ and so $q$ is a common factor in its power series expansion. Consequently,

$$
\left\|f^{\odot} \varphi\right\|_{2}^{2}=|f(0)|^{2}+\left\|\varphi *\left((T f)^{\odot} \varphi\right)\right\|_{2}^{2} .
$$

By Lemma 6.4. we have

$$
\left\|\varphi *\left((T f)^{\odot} \varphi\right)\right\|_{2} \leq\|\varphi\|_{\infty}\left\|(T f)^{\odot} \varphi\right\|_{2} \leq\left\|(T f)^{\odot} \varphi\right\|_{2},
$$

and hence

$$
\left\|f^{\odot} \varphi\right\|_{2}^{2} \leq|f(0)|^{2}+\left\|(T f)^{\odot} \varphi\right\|_{2}^{2} .
$$

Now successively substituting $T f, T^{2} f, \cdots, T^{n} f$ for $f$ in (6.4) yields

$$
\begin{gathered}
\left\|(T f)^{\odot} \varphi\right\|_{2}^{2} \leq|(T f)(0)|^{2}+\left\|\left(T^{2} f\right)^{\odot} \varphi\right\|_{2}^{2}, \\
\left\|\left(T^{2} f\right)^{\odot} \varphi\right\|_{2}^{2} \leq\left|\left(T^{2} f\right)(0)\right|^{2}+\left\|\left(T^{3} f\right)^{\odot} \varphi\right\|_{2}^{2}, \\
\vdots \\
\left\|\left(T^{n} f\right)^{\odot} \varphi\right\|_{2}^{2} \leq\left|\left(T^{n} f\right)(0)\right|^{2}+\left\|\left(T^{n+1} f\right)^{\odot} \varphi\right\|_{2}^{2} .
\end{gathered}
$$

Putting all these inequalities together, we get

$$
\left\|f^{\odot} \varphi\right\|_{2}^{2} \leq \sum_{k=0}^{n}\left|\left(T^{k} f\right)(0)\right|^{2}+\left\|\left(T^{n+1} f\right)^{\odot} \varphi\right\|_{2}^{2} .
$$

for any $n \in \mathbb{N}$.

Now recall that $f$ is a polynomial. If we choose $n$ be the degree of $f$, then $T^{n+1} f$ vanishes identically and so does $\left(T^{n+1} f\right)^{\odot} \varphi$. It follows from (6.3) and (6.5) that

$$
\left\|f^{\odot} \varphi\right\|_{2}^{2} \leq \sum_{k=0}^{n}\left|\left(T^{k} f\right)(0)\right|^{2}=\sum_{k=0}^{n}|\hat{f}(k)|^{2}=\|f\|_{2}^{2},
$$

which shows that $\left\|f^{\odot} \varphi\right\|_{2} \leq\|f\|_{2}$ holds at least for any regular polynomials.

Now we consider the general case that $f \in H^{2}(\mathbb{B})$. Let $f_{n}$ be the $n$-th partial sum of the power series expansion of $f$, i.e.

$$
f_{n}(q)=\sum_{k=0}^{n} q^{k} \hat{f}(k)
$$

Then $f_{n}$ is a polynomial and

$$
\left\|f_{n}-f\right\|_{2}^{2}=\sum_{k=n+1}^{\infty}|\hat{f}(k)|^{2} \longrightarrow 0 \quad \text { as } n \longrightarrow 0 .
$$


By (6.6), for all $n \in \mathbb{N}$,

$$
\left\|f_{n}^{\odot} \varphi\right\|_{2} \leq\left\|f_{n}\right\|_{2} \leq\|f\|_{2} .
$$

Notice that $|\varphi(q)| \leq|q|$ in virtue of Theorem 2.16. Using the arguments similar to those used in Remark 4.2 and in Lemma 6.2 we obtain that for any fixed $r \in(0,1)$,

$$
\sup _{|q| \leq r}\left|f_{n}^{\odot} \varphi(q)-f^{\odot} \varphi(q)\right| \leq\left(\frac{1}{1-r^{2}}\right)^{\frac{1}{2}}\|f\|_{2} \longrightarrow 0 \quad \text { as } n \longrightarrow \infty,
$$

which implies that $\left\{f_{n}^{\odot} \varphi\right\}_{n \in \mathbb{N}}$ converges uniformly to $f^{\odot} \varphi$ on every compact subset $U \subset \mathbb{B}$. Consequently, for any $r \in(0,1)$ and $I \in \mathbb{S}$, we have

$$
\begin{aligned}
\left(\frac{1}{2 \pi} \int_{-\pi}^{\pi}\left|f^{\odot} \varphi\left(r e^{I \theta}\right)\right|^{2} d \theta\right)^{\frac{1}{2}} & =\lim _{n \rightarrow \infty}\left(\frac{1}{2 \pi} \int_{-\pi}^{\pi}\left|f_{n}^{\odot} \varphi\left(r e^{I \theta}\right)\right|^{2} d \theta\right)^{\frac{1}{2}} \\
& \leq \limsup _{n \longrightarrow \infty}\left\|f_{n}^{\odot} \varphi\right\|_{2} \\
& \leq \limsup _{n \longrightarrow \infty}\left\|f_{n}\right\|_{2} \\
& \leq\|f\|_{2},
\end{aligned}
$$

which implies that $f \odot^{\odot} \varphi \in H^{2}(\mathbb{B})$ and $\left\|f^{\odot} \varphi\right\|_{2} \leq\|f\|_{2}$. In the second inequality we have used Proposition 2.18. The penultimate and last equations follow by (6.7).

As for $f_{\odot} \varphi$, we can prove the result in the very same manner, it suffices to notice that

and hence

$$
f(q)=f(0)+q(T f)(q)=f(0)+(T f)(q) * q, \quad \forall q \in \mathbb{B},
$$

$$
f_{\odot} \varphi(q)=f(0)+((T f) \odot \varphi) * \varphi(q), \quad \forall q \in \mathbb{B} .
$$

An alternative easier method is the conjugate method as in Lemma 6.4, which is the following

$$
\left\|f_{\odot} \varphi\right\|_{2}=\left\|(f \odot \varphi)^{c}\right\|_{2}=\left\|f^{c} \odot \varphi^{c}\right\|_{2} \leq\left\|f^{c}\right\|_{2}=\|f\|_{2} .
$$

In the second equation we have used equation (4.4). Now the proof is completed.

Let $\varphi: \mathbb{B} \rightarrow \mathbb{B}$ be a regular function and $\varphi(0)=0$, then $\varphi$ induces two composition operators $C_{\varphi}$ and $D_{\varphi}$ on $H^{2}(\mathbb{B})$ defined by

$$
C_{\varphi}(f)=f^{\odot} \varphi, \quad D_{\varphi}(f)=f_{\odot} \varphi .
$$

Obviously, $C_{\varphi}$ is a right $\mathbb{H}$-linear operator while $D_{\varphi}$ a left $\mathbb{H}$-linear operator on Hilbert $\mathbb{H}$-module $H^{2}(\mathbb{B})$. Namely,

$$
C_{\varphi}(f * \lambda+g * \mu)=C_{\varphi}(f) * \lambda+C_{\varphi}(g) * \mu
$$

and

$$
D_{\varphi}(\lambda * f+\mu * g)=\lambda * D_{\varphi}(f)+\mu * D_{\varphi}(g)
$$

for all $\lambda, \mu \in \mathbb{H}$ and $f, g \in H^{2}(\mathbb{B})$. Moreover, Theorem 6.1 shows that $C_{\varphi}$ and $D_{\varphi}$ are two bounded composition operators.

Corollary 6.6. Let $\varphi: \mathbb{B} \rightarrow \mathbb{B}$ be a regular function and $\varphi(0)=0$, then $C_{\varphi}$ and $D_{\varphi}$ are two bounded composition operators on $H^{2}(\mathbb{B})$ with norms $\left\|C_{\varphi}\right\|=\left\|D_{\varphi}\right\|=1$.

Proof. On one hand, it follows from Theorem 6.1 that $\left\|C_{\varphi}\right\| \leq 1$ and $\left\|D_{\varphi}\right\| \leq 1$. On the other hand, $C_{\varphi}(1)=D_{\varphi}(1)=1$, which implies that $\left\|C_{\varphi}\right\| \geq 1$ and $\left\|D_{\varphi}\right\| \geq 1$. Consequently, $\left\|C_{\varphi}\right\|=\left\|D_{\varphi}\right\|=1$. This completes the proof of Theorem 1.1. 


\section{BOUNDEDNESS OF COMPOSITION OPERATORS}

The boundedness of the slice composition operators $C_{\varphi}$ and $D_{\varphi}$ on $H^{p}(\mathbb{B})$ are studied in this section.

Let $\varphi: \mathbb{B} \rightarrow \mathbb{B}$ be a regular function. It induces operators $C_{\varphi}$ and $D_{\varphi}$ via

$$
C_{\varphi}(f)=f^{\odot} \varphi, \quad D_{\varphi}(f)=f_{\odot} \varphi .
$$

We shall show that in $H^{2}(\mathbb{B})$ we have

$$
\left\|C_{\varphi}\right\|=\left\|D_{\varphi}\right\|=\left(\frac{1+|\varphi(0)|}{1-|\varphi(0)|}\right)^{\frac{1}{2}}
$$

for any regular Möbius transformation $\varphi$ of $\mathbb{B}$.

We first consider the variant of Theorem 6.1 without the restriction $\varphi(0)=0$.

Theorem 7.1. Let $\varphi: \mathbb{B} \rightarrow \mathbb{B}$ be a regular function such that $\varphi\left(\mathbb{B}_{I}\right) \subseteq \mathbb{B}_{I}$ for some $I \in \mathbb{S}$, then

$$
\left\|f^{\odot} \varphi\right\|_{2} \leq\left(\frac{1+|\varphi(0)|}{1-|\varphi(0)|}\right)^{\frac{1}{2}}\|f\|_{2}, \quad\left\|f_{\odot} \varphi\right\|_{2} \leq\left(\frac{1+|\varphi(0)|}{1-|\varphi(0)|}\right)^{\frac{1}{2}}\|f\|_{2}
$$

for any $f \in H^{2}(\mathbb{B})$.

Proof. Consider the power series expansion of $f$

$$
f(q)=\sum_{n=0}^{\infty} q^{n} \hat{f}(n)
$$

Taking $J \in \mathbb{S}$ with $J \perp I$, we can decompose $\hat{f}(n)$ in the form

$$
\hat{f}(n)=\hat{g}(n)+\hat{h}(n) J
$$

where $\hat{g}(n), \hat{h}(n) \in \mathbb{C}_{I}$ for all $n \in \mathbb{N}$. Consequently,

$$
f(q)=g(q)+h(q) J
$$

and

$$
\|f\|_{2}^{2}=\|g\|_{2}^{2}+\|h\|_{2}^{2}
$$

where $g(q)=\sum_{n=0}^{\infty} q^{n} \hat{g}(n)$ and $h(q)=\sum_{n=0}^{\infty} q^{n} \hat{h}(n)$ are two regular functions on $\mathbb{B}$ such that each of them maps $\mathbb{B}_{I}$ into itself respectively. By assumption, $\varphi\left(\mathbb{B}_{I}\right) \subseteq \mathbb{B}_{I}$, so that

$$
\begin{aligned}
\left(f_{\odot} \varphi\right)_{I}(z) & =\sum_{n=0}^{\infty}(\hat{g}(n)+\hat{h}(n) J) * \varphi_{I}^{n}(z) \\
& =\sum_{n=0}^{\infty} \varphi_{I}^{n}(z) \hat{g}(n)+\sum_{n=0}^{\infty}\left(\overline{\varphi_{I}(\bar{z})}\right)^{n} \hat{h}(n) J \\
& =g_{I} \circ \varphi_{I}(z)+h_{I} \circ \overline{\varphi_{I}(\bar{z})} J .
\end{aligned}
$$

In the penultimate equation we have used the fact that $J * \psi(z)=\overline{\psi(\bar{z})} J$ for any holomorphic function $\psi$ in the variable $z \in \mathbb{C}_{I}$ and $I, J \in \mathbb{S}$ with $I \perp J$, which can be obtained from the definition of $*$-product. 
Now it follows from the classical theory of composition operators on the Hardy space $H^{2}$ (cf. Corollary 3.7 in [8]) and Proposition 2.18 that

$$
\begin{aligned}
\left\|f_{\odot} \varphi\right\|_{2}^{2}=\left\|\left(f_{\odot} \varphi\right)_{I}\right\|_{2}^{2} & =\left\|g_{I} \circ \varphi_{I}\right\|_{2}^{2}+\left\|h_{I} \circ \overline{\varphi_{I}(\cdot)}\right\|_{2}^{2} \\
& \leq \frac{1+|\varphi(0)|}{1-|\varphi(0)|}\left(\left\|g_{I}\right\|_{2}^{2}+\left\|h_{I}\right\|_{2}^{2}\right) \\
& =\frac{1+|\varphi(0)|}{1-|\varphi(0)|}\left(\|g\|_{2}^{2}+\|h\|_{2}^{2}\right) \\
& =\frac{1+|\varphi(0)|}{1-|\varphi(0)|}\|f\|_{2}^{2} .
\end{aligned}
$$

As for $f^{\odot} \varphi$, we can prove the result in the very same manner, it suffices to notice that

$$
\left(f^{\odot} \varphi\right)_{I}(z)=g_{I} \circ \varphi_{I}(z)+h_{I} \circ \varphi_{I}(z) J
$$

As in Theorem 6.1] an alternative easier method is the conjugate method, which is the following

$\left\|f^{\odot} \varphi\right\|_{2}=\left\|\left(f^{\odot} \varphi\right)^{c}\right\|_{2}=\left\|f_{\odot}^{c} \varphi^{c}\right\|_{2} \leq\left(\frac{1+\left|\varphi^{c}(0)\right|}{1-\left|\varphi^{c}(0)\right|}\right)^{\frac{1}{2}}\left\|f^{c}\right\|_{2}=\left(\frac{1+|\varphi(0)|}{1-|\varphi(0)|}\right)^{\frac{1}{2}}\|f\|_{2}$.

In the second equation we have used equation (4.3). Now the proof is completed.

Corollary 7.2. Let $\varphi: \mathbb{B} \rightarrow \mathbb{B}$ be a regular function such that $\varphi\left(\mathbb{B}_{I}\right) \subseteq \mathbb{B}_{I}$ for some $I \in \mathbb{S}$, then $C_{\varphi}$ and $D_{\varphi}$ are two bounded composition operators on $H^{2}(\mathbb{B})$. Moreover,

and

$$
\left(\frac{1}{1-|\varphi(0)|^{2}}\right)^{\frac{1}{2}} \leq\left\|C_{\varphi}\right\| \leq\left(\frac{1+|\varphi(0)|}{1-|\varphi(0)|}\right)^{\frac{1}{2}}
$$

$$
\left(\frac{1}{1-|\varphi(0)|^{2}}\right)^{\frac{1}{2}} \leq\left\|D_{\varphi}\right\| \leq\left(\frac{1+|\varphi(0)|}{1-|\varphi(0)|}\right)^{\frac{1}{2}} .
$$

Proof. The two upper bounds follow from Theorem 7.1. To prove the two lower bounds, consider the restriction $C_{\varphi_{I}}$ of $C_{\varphi}$ to $H^{2}\left(\mathbb{B}_{I}\right)$ and its adjoint $C_{\varphi_{I}}^{*}$ acting on the evaluation kernels $K_{w}$ in $H^{2}\left(\mathbb{B}_{I}\right)$, then by the classical $H^{2}$ theory (see for instance Corollary 2.11 in $[8$ ), we have

$$
C_{\varphi_{I}}^{*}\left(K_{w}\right)=K_{\varphi_{I}(w)}
$$

and

$$
K_{w}(z)=\frac{1}{1-\bar{w} z} \quad \text { with } \quad\left\|K_{w}\right\|_{2}=\left(\frac{1}{1-|w|^{2}}\right)^{\frac{1}{2}}
$$

for all $z, w \in \mathbb{B}_{I}$. It is easy to prove that

$$
\left\|C_{\varphi}\right\|=\left\|C_{\varphi_{I}}\right\| .
$$

Now it follows from (7.1) and (7.2) that

$$
\left\|C_{\varphi}\right\|=\left\|C_{\varphi_{I}}\right\|=\left\|C_{\varphi_{I}}^{*}\right\| \geq \frac{\left\|K_{\varphi_{I}(w)}\right\|_{2}}{\left\|K_{w}\right\|_{2}}=\left(\frac{1-|w|^{2}}{1-\left|\varphi_{I}(w)\right|^{2}}\right)^{\frac{1}{2}} .
$$

Taking $w=0$ yields that

$$
\left\|C_{\varphi}\right\| \geq\left(\frac{1}{1-|\varphi(0)|^{2}}\right)^{\frac{1}{2}}
$$


It follows from 4.3 that

$$
\left(D_{\varphi}(f)\right)^{c}=C_{\varphi^{c}}\left(f^{c}\right),
$$

from which we can easily prove that

$$
\left\|D_{\varphi}\right\|=\left\|C_{\varphi^{c}}\right\|
$$

Consequently,

$$
\left\|D_{\varphi}\right\| \geq\left(\frac{1}{1-\left|\varphi^{c}(0)\right|^{2}}\right)^{\frac{1}{2}}=\left(\frac{1}{1-|\varphi(0)|^{2}}\right)^{\frac{1}{2}}
$$

which completes the proof.

If $\varphi$ is a regular Möbius transformation $\varphi_{a}$ of $\mathbb{B}$ (see [25]), i.e.

$$
\varphi_{a}(q)=(1-q \bar{a})^{-*} *(a-q)=(a-q) *(1-q \bar{a})^{-*}=a-\left(1-|a|^{2}\right) \sum_{n=1}^{\infty} q^{n} \bar{a}^{n-1}
$$

for some $a \in \mathbb{B}$, we can obtain more precise result, which is as follows.

Theorem 7.3. Let $\varphi$ be a regular Möbius transformation of $\mathbb{B}$, then the composition operators $C_{\varphi}$ and $D_{\varphi}$ are bounded on $H^{2}(\mathbb{B})$ with norms

$$
\left\|C_{\varphi}\right\|=\left\|D_{\varphi}\right\|=\left(\frac{1+|\varphi(0)|}{1-|\varphi(0)|}\right)^{\frac{1}{2}}
$$

Proof. Let $\varphi(q)=(1-q \bar{a})^{-*} *(a-q)=(a-q) *(1-q \bar{a})^{-*}$ for some $a \in \mathbb{B}_{I}$, then $\varphi\left(\mathbb{B}_{I}\right)=\mathbb{B}_{I}$, it follows from Corollary 7.2 that

$$
\left\|C_{\varphi}(f)\right\|_{2} \leq\left(\frac{1+|\varphi(0)|}{1-|\varphi(0)|}\right)^{\frac{1}{2}}\|f\|_{2}
$$

and

$$
\left\|D_{\varphi}(f)\right\|_{2} \leq\left(\frac{1+|\varphi(0)|}{1-|\varphi(0)|}\right)^{\frac{1}{2}}\|f\|_{2},
$$

which are the two upper inequalities. Now we show that each of them is sharp respectively. An easy calculation gives

$$
1-\left|\varphi_{I}(w)\right|^{2}=\frac{\left(1-|a|^{2}\right)\left(1-|w|^{2}\right)}{|1-w \bar{a}|^{2}},
$$

Substituting (7.7) into (7.3) yields

$$
\left\|C_{\varphi}\right\| \geq\left(\frac{|1-w \bar{a}|^{2}}{1-|a|^{2}}\right)^{\frac{1}{2}}, \quad \forall w \in \mathbb{B}_{I}
$$

Now if $a=|a| e^{I \theta}$, we take $w=-r e^{I \theta}$ so that

$$
\left\|C_{\varphi}\right\| \geq \lim _{r \rightarrow 1^{-}}\left(\frac{|1-w \bar{a}|^{2}}{1-|a|^{2}}\right)^{\frac{1}{2}}=\left(\frac{1+|a|}{1-|a|}\right)^{\frac{1}{2}}=\left(\frac{1+|\varphi(0)|}{1-|\varphi(0)|}\right)^{\frac{1}{2}}
$$

which implies that inequality (7.5) is sharp and

$$
\left\|C_{\varphi}\right\|=\left(\frac{1+|\varphi(0)|}{1-|\varphi(0)|}\right)^{\frac{1}{2}}
$$

It follows from (4.3) that

$$
\left(D_{\varphi}(f)\right)^{c}=C_{\varphi^{c}}\left(f^{c}\right)
$$


from which we can easily prove that

$$
\left\|D_{\varphi}\right\|=\left\|C_{\varphi^{c}}\right\|=\left(\frac{1+\left|\varphi^{c}(0)\right|}{1-\left|\varphi^{c}(0)\right|}\right)^{\frac{1}{2}}=\left(\frac{1+|\varphi(0)|}{1-|\varphi(0)|}\right)^{\frac{1}{2}} .
$$

Notice that $\varphi\left(\mathbb{B}_{I}\right) \subseteq \mathbb{B}_{I}$, the very definition of regular composition and the identity principle 2.14 allow us to conclude that

$$
f^{\odot} \varphi=\operatorname{ext}\left(f_{I} \circ \varphi_{I}\right)
$$

and hence

$\left(f^{\odot} \varphi\right)^{\odot} \varphi=\operatorname{ext}\left(\left(f^{\odot} \varphi\right)_{I} \circ \varphi_{I}\right)=\operatorname{ext}\left(\left(f_{I} \circ \varphi_{I}\right) \circ \varphi_{I}\right)=\operatorname{ext}\left(f_{I} \circ\left(\varphi_{I} \circ \varphi_{I}\right)\right)=\operatorname{ext}\left(f_{I}\right)=f$.

Namely,

$$
C_{\varphi}\left(C_{\varphi}(f)\right)=f
$$

Consequently,

$$
\|f\|_{2}=\left\|C_{\varphi}\left(C_{\varphi}(f)\right)\right\|_{2} \leq\left\|C_{\varphi}\right\|\left\|C_{\varphi}(f)\right\|_{2}
$$

that is,

$$
\left\|C_{\varphi}(f)\right\|_{2} \geq \frac{1}{\left\|C_{\varphi}\right\|}\|f\|_{2}=\left(\frac{1-|\varphi(0)|}{1+|\varphi(0)|}\right)^{\frac{1}{2}}\|f\|_{2} .
$$

Similarly, we can prove that $D_{\varphi}\left(D_{\varphi}(f)\right)=f$, which implies that

$$
\|f\|_{2} \leq\left\|D _ { \varphi } \left|\left\||| D_{\varphi}(f)\right\|_{2}\right.\right.
$$

and hence

$$
\left\|D_{\varphi}(f)\right\|_{2} \geq \frac{1}{\left\|D_{\varphi}\right\|}\|f\|_{2}=\left(\frac{1-|\varphi(0)|}{1+|\varphi(0)|}\right)^{\frac{1}{2}}\|f\|_{2} .
$$

Finally, Let $\left\{f_{n}\right\}$ be a sequence that exhibits the norm of $C_{\varphi}$, then the substitution $g_{n}=C_{\varphi} g_{n}$ shows that the inequality (7.8) is sharp. Similarly, we can prove that the inequality (7.9) is sharp also.

As a result, we have proved that

$$
\left(\frac{1-|\varphi(0)|}{1+|\varphi(0)|}\right)^{\frac{1}{2}}\|f\|_{2} \leq\left\|C_{\varphi}(f)\right\|_{2} \leq\left(\frac{1+|\varphi(0)|}{1-|\varphi(0)|}\right)^{\frac{1}{2}}\|f\|_{2}
$$

and

$$
\left(\frac{1-|\varphi(0)|}{1+|\varphi(0)|}\right)^{\frac{1}{2}}\|f\|_{2} \leq\left\|D_{\varphi}(f)\right\|_{2} \leq\left(\frac{1+|\varphi(0)|}{1-|\varphi(0)|}\right)^{\frac{1}{2}}\|f\|_{2} .
$$

Moreover, these inequalities are best possible.

Now we consider the $H^{p}(\mathbb{B})$ version of Theorem 6.1.

Theorem 7.4. Let $\varphi: \mathbb{B} \rightarrow \mathbb{B}$ be a regular function such that $\varphi\left(\mathbb{B}_{I}\right) \subseteq \mathbb{B}_{I}$ for some $I \in \mathbb{S}$, then $C_{\varphi}$ and $D_{\varphi}$ are two bounded composition operators on $H^{p}(\mathbb{B})$ for any $1 \leq p<\infty$. Moreover,

$$
\left\|C_{\varphi}\right\|_{p} \simeq\left\|D_{\varphi}\right\|_{p} \simeq\left(1-|\varphi(0)|^{2}\right)^{-\frac{1}{p}}
$$


Proof. First applying the classical theory of composition operator on the Hardy space $H^{p}$ (cf. Corollary 3.7 in [8]) to $f_{I} \circ \varphi_{I}$ yields

$$
\left(\frac{1}{1-|\varphi(0)|^{2}}\right)^{\frac{1}{p}}\left\|f_{I}\right\|_{p} \leq\left\|f_{I} \circ \varphi_{I}\right\|_{p} \leq\left(\frac{1+|\varphi(0)|}{1-|\varphi(0)|}\right)^{\frac{1}{p}}\left\|f_{I}\right\|_{p}
$$

for any $f \in H^{p}(\mathbb{B})$. Taking into account the fundamental convexity equality

$$
(a+b)^{p} \leq 2^{p-1}\left(a^{p}+b^{p}\right)
$$

for any $a, b \in[0, \infty)$ and $p \geq 1$, we can easily prove that

$$
\left\|f_{I}\right\|_{p} \leq\|f\|_{p} \leq 2^{2-\frac{1}{p}}\left\|f_{I}\right\|_{p}
$$

and

$$
\left\|f^{c}\right\|_{p} \leq 2^{\frac{1}{p}}\|f\|_{p}
$$

On one hand, it follows from (7.10), (7.11) and the fact that $\left(C_{\varphi}(f)\right)_{I}=f_{I} \circ \varphi_{I}$ that

$$
\left\|C_{\varphi}(f)\right\|_{p} \leq 2^{2-\frac{1}{p}}|| f_{I} \circ \varphi_{I}\left\|_{p} \leq 2^{2-\frac{1}{p}}\left(\frac{1+|\varphi(0)|}{1-|\varphi(0)|}\right)^{\frac{1}{p}}\right\| f \|_{p},
$$

which together with (7.12) give

$$
\begin{aligned}
\left\|D_{\varphi}(f)\right\|_{p} & \leq 2^{\frac{1}{p}}\left\|\left(D_{\varphi}(f)\right)^{c}\right\|_{p} \\
& =2^{\frac{1}{p}}\left\|C_{\varphi^{c}}\left(f^{c}\right)\right\|_{p} \\
& \leq 4\left(\frac{1+|\varphi(0)|}{1-|\varphi(0)|}\right)^{\frac{1}{p}}\left\|f^{c}\right\|_{p} \\
& \leq 2^{2+\frac{1}{p}}\left(\frac{1+|\varphi(0)|}{1-|\varphi(0)|}\right)^{\frac{1}{p}}\|f\|_{p}
\end{aligned}
$$

for any $f \in H^{p}(\mathbb{B})$. On the other hand,

$$
\left\|C_{\varphi}(f)\right\|_{p}=\left\|\left(C_{\varphi}(f)\right)_{I}\right\|_{p}=\left\|f_{I} \circ \varphi_{I}\right\|_{p} \geq 2^{-\left(2-\frac{1}{p}\right)}\left(\frac{1}{1-|\varphi(0)|^{2}}\right)^{\frac{1}{p}}\|f\|_{p},
$$

which implies that

$$
\begin{aligned}
\left\|D_{\varphi}(f)\right\|_{p} & \geq 2^{-\frac{1}{p}}\left\|\left(D_{\varphi}(f)\right)^{c}\right\|_{p} \\
& =2^{-\frac{1}{p}}\left\|C_{\varphi^{c}}\left(f^{c}\right)\right\|_{p} \\
& \geq \frac{1}{4}\left(\frac{1}{1-|\varphi(0)|^{2}}\right)^{\frac{1}{p}}\left\|f^{c}\right\|_{p} \\
& \geq 2^{-\left(2+\frac{1}{p}\right)}\left(\frac{1}{1-|\varphi(0)|^{2}}\right)^{\frac{1}{p}}\|f\|_{p}
\end{aligned}
$$

for any $f \in H^{p}(\mathbb{B})$.

To be concluded, we have proved

$$
2^{-\left(2-\frac{1}{p}\right)}\left(\frac{1}{1-|\varphi(0)|^{2}}\right)^{\frac{1}{p}} \leq\left\|C_{\varphi}\right\| \leq 2^{2-\frac{1}{p}}\left(\frac{1+|\varphi(0)|}{1-|\varphi(0)|}\right)^{\frac{1}{p}}
$$


and

$$
2^{-\left(2+\frac{1}{p}\right)}\left(\frac{1}{1-|\varphi(0)|^{2}}\right)^{\frac{1}{p}} \leq\left\|D_{\varphi}\right\| \leq 2^{2+\frac{1}{p}}\left(\frac{1+|\varphi(0)|}{1-|\varphi(0)|}\right)^{\frac{1}{p}}
$$

as desired.

We remark that the same arguments as in the proof of the preceding theorem leads to the following result. More precisely, let $\varphi$ is a regular Möbius transformation of $\mathbb{B}$, then $C_{\varphi}$ and $D_{\varphi}$ are two bounded composition operators on $H^{p}(\mathbb{B})(1 \leq p<\infty)$. Moreover,

$$
2^{-\left(2-\frac{1}{p}\right)}\left(\frac{1-|\varphi(0)|}{1+|\varphi(0)|}\right)^{\frac{1}{p}}\|f\|_{p} \leq\left\|C_{\varphi}(f)\right\|_{p} \leq 2^{2-\frac{1}{p}}\left(\frac{1+|\varphi(0)|}{1-|\varphi(0)|}\right)^{\frac{1}{p}}\|f\|_{p}
$$

and

$$
2^{-\left(2+\frac{1}{p}\right)}\left(\frac{1-|\varphi(0)|}{1+|\varphi(0)|}\right)^{\frac{1}{p}}\|f\|_{p} \leq\left\|D_{\varphi}(f)\right\|_{p} \leq 2^{2+\frac{1}{p}}\left(\frac{1+|\varphi(0)|}{1-|\varphi(0)|}\right)^{\frac{1}{p}}\|f\|_{p} .
$$

\section{COMPACTNESS OF COMPOSITION OPERATORS}

The compactness of composition operators $C_{\varphi}$ and $D_{\varphi}$ on $H^{p}(\mathbb{B})$ is studied in this section.

We need to specify precisely how much the inducing map $\varphi$ has to compress the unit ball into itself in order to insure that the operators $C_{\varphi}$ and $D_{\varphi}$ compress bounded subsets of $H^{p}(\mathbb{B})$ into relatively compact ones. The most drastic way $\varphi$ can compress the unit ball is to take it to a point, in which case the resulting composition operators $C_{\varphi}$ and $D_{\varphi}$ have one dimensional range (the space of constant functions), and are therefore compact. The next result shows that this compactness persists if we merely assume that $\varphi(\mathbb{B})$ is relatively compact in $\mathbb{B}$.

Theorem 8.1. Let $\varphi: \mathbb{B} \rightarrow \mathbb{B}$ be a regular function on $\mathbb{B}$ such that $\|\varphi\|_{\infty}<1$. Then both $C_{\varphi}$ and $D_{\varphi}$ are compact operators on $H^{p}(\mathbb{B})$ for any $1 \leq p \leq \infty$.

Proof. In the case that $p=2$, the result can be easily proved due to the speciality of $H^{2}(\mathbb{B})$. For each positive integer $N$ define the operator $S_{N}: H^{2}(\mathbb{B}) \rightarrow H^{2}(\mathbb{B})$ via

$$
S_{N} f(q)=\sum_{n=0}^{N} \varphi^{* n} \hat{f}(n)
$$

for any $f \in H^{2}(\mathbb{B})$ with the Taylor expansion

$$
f(q)=\sum_{n=0}^{\infty} q^{n} \hat{f}(n) \in H^{2}(\mathbb{B}) .
$$

Thus $S_{N}$ maps $H^{2}(\mathbb{B})$ onto the right $\mathbb{H}$-linear span of the first $n$ regular powers of $C_{\varphi}$. Obviously, $S_{N}$ is a bounded, finite rank operator on $H^{2}(\mathbb{B})$. We claim that

$$
\left\|S_{N}-C_{\varphi}\right\|_{2} \longrightarrow 0 \text { as } n \longrightarrow \infty .
$$


This follows from the calculation below:

$$
\begin{aligned}
\left\|\left(S_{N}-C_{\varphi}\right) f\right\|_{2} & =\left\|\sum_{n=N+1}^{\infty} \varphi^{* n} \hat{f}(n)\right\|_{2} \leq \sum_{n=N+1}^{\infty}|\hat{f}(n)|\left\|\varphi^{* n}\right\|_{2} \\
& \leq \sum_{n=N+1}^{\infty}|\hat{f}(n)|\|\varphi\|_{\infty}^{n} \leq\left(\sum_{n=N+1}^{\infty}|\hat{f}(n)|^{2}\right)^{\frac{1}{2}}\left(\sum_{n=N+1}^{\infty}\|\varphi\|_{\infty}^{2 n}\right)^{\frac{1}{2}} \\
& =\frac{\|\varphi\|_{\infty}^{N+1}}{\left(1-\|\varphi\|_{\infty}^{2}\right)^{\frac{1}{2}}}\|f\|_{2} .
\end{aligned}
$$

Thus

$$
\left\|S_{N}-C_{\varphi}\right\|_{2} \leq \frac{\|\varphi\|_{\infty}^{N+1}}{\left(1-\|\varphi\|_{\infty}^{2}\right)^{\frac{1}{2}}} \longrightarrow 0 \quad \text { as } n \longrightarrow \infty .
$$

This exhibits $C_{\varphi}$ as an operator norm limit of finite rank operators, so it is compact on $H^{2}(\mathbb{B})$. Similarly, it turns out that $D_{\varphi}$ is compact on $H^{2}(\mathbb{B})$ as well.

To prove Theorem 8.1 for $p \neq 2$, we need an $H^{p}(\mathbb{B})$ version of Lemma 6.2.

Lemma 8.2. Let $f \in H^{p}(\mathbb{B})$ with $1 \leq p<\infty$, then for any $q \in \mathbb{B}$,

$$
|f(q)| \leq \sqrt{2}\left(\frac{1}{1-|q|^{2}}\right)^{\frac{1}{p}}\|f\|_{p}
$$

Proof. For any fixed $I \in \mathbb{S}$, we apply the splitting lemma to write

$$
f_{I}(z)=F(z)+G(z) J,
$$

where $F, G: \mathbb{B}_{I} \rightarrow \mathbb{C}_{I}$ are two holomorphic functions and $J \in \mathbb{S}$ with $J \perp I$. Then by the very definition, $F, G \in H^{p}\left(\mathbb{B}_{I}\right)$. Moreover, $\|F\|_{p} \leq\|f\|_{p}$ and $\|G\|_{p} \leq\|f\|_{p}$. Applying the classical result(cf. Corollary 2.14 in [8]) from complex analysis to $F$ and $G$ yields

$$
|F(z)| \leq\left(\frac{1}{1-|z|^{2}}\right)^{\frac{1}{p}}\|F\|_{p}, \quad|G(z)| \leq\left(\frac{1}{1-|z|^{2}}\right)^{\frac{1}{p}}\|G\|_{p}
$$

Therefore,

$$
|f(z)|^{2}=|F(z)|^{2}+|G(z)|^{2} \leq\left(\frac{1}{1-|z|^{2}}\right)^{\frac{2}{p}}\left(\|F\|_{p}^{2}+\|G\|_{p}^{2}\right) \leq 2\left(\frac{1}{1-|z|^{2}}\right)^{\frac{2}{p}}\|f\|_{p}^{2}
$$

as desired.

This leads to an $H^{p}(\mathbb{B})$ version of the Cauchy inequality.

Lemma 8.3. Let $f \in H^{p}(\mathbb{B})$ with $1 \leq p \leq \infty$, then

$$
\left|f^{(n)}(0)\right| \leq \sqrt{2} n ! e^{\frac{1}{p}}\left(1+\frac{n p}{2}\right)^{\frac{1}{p}}\|f\|_{p} \quad \text { for } \quad 1 \leq p<\infty,
$$

and

$$
\left|f^{(n)}(0)\right| \leq n !\|f\|_{p} \quad \text { for } \quad p=\infty .
$$

Proof. When $p=\infty$, the result is the Cauchy inequality.

When $1 \leq p<\infty$, for any $I \in \mathbb{S}$ and $r \in(0,1)$, it follows from the Cauchy integral formula that

$$
f^{(n)}(0)=\frac{n !}{2 \pi I} \int_{\partial B_{I}(0, r)} \frac{d z}{z^{n+1}} f(z),
$$


which together with Lemma 8.2 implies that

$$
\left|f^{(n)}(0)\right| \leq \sqrt{2} \frac{n !}{r^{n}}\left(\frac{1}{1-r^{2}}\right)^{\frac{1}{p}}\|f\|_{p}, \quad \forall r \in(0,1) .
$$

Taking $r=\left(\frac{n p}{n p+2}\right)^{\frac{1}{2}}$ yields

$$
\left|f^{(n)}(0)\right| \leq \sqrt{2} n !\left(1+\frac{n p}{2}\right)^{\frac{1}{p}}\left(1+\frac{2}{n p}\right)^{\frac{n}{2}}\|f\|_{p} \leq \sqrt{2} n ! e^{\frac{1}{p}}\left(1+\frac{n p}{2}\right)^{\frac{1}{p}}\|f\|_{p}
$$

Finally, we come to prove Theorem 8.1 for the remaining case for $p \neq 2$.

Proof. Assume that $p \neq 2$. For each positive integer $N$ define the operator $S_{N}$ : $H^{p}(\mathbb{B}) \rightarrow H^{p}(\mathbb{B})$ via

$$
S_{N} f(q)=\sum_{n=0}^{N} \varphi^{* n} \frac{f^{(n)}(0)}{n !}
$$

for any $f \in H^{p}(\mathbb{B})$ with the Taylor expansion

$$
f(q)=\sum_{n=0}^{\infty} q^{n} \frac{f^{(n)}(0)}{n !} .
$$

Thus $S_{N}$ is a bounded, finite rank operator on $H^{p}(\mathbb{B})$ and $\left\|S_{N}\right\|_{p}$ at most

$$
\sqrt{2} e^{\frac{1}{p}} \sum_{n=0}^{N}\left(1+\frac{n p}{2}\right)^{\frac{1}{p}}\|\varphi\|_{\infty}^{n}
$$

for $p<\infty$, and

$$
\sum_{n=0}^{N}\|\varphi\|_{\infty}^{n}=\frac{1-\|\varphi\|_{\infty}^{N+1}}{1-\|\varphi\|_{\infty}}
$$

for $p=\infty$.

To show $S_{N}$ is compact, it suffices to prove that

$$
\left\|S_{N}-C_{\varphi}\right\|_{p} \longrightarrow 0 \text { as } n \longrightarrow \infty
$$

which follows from the inequalities

$$
\left\|S_{N}-C_{\varphi}\right\|_{p} \leq \sqrt{2} e^{\frac{1}{p}} \sum_{n=N}^{\infty}\left(1+\frac{n p}{2}\right)^{\frac{1}{p}}\|\varphi\|_{\infty}^{n}, \quad p<\infty
$$

and

$$
\left\|S_{N}-C_{\varphi}\right\|_{\infty} \leq \frac{\|\varphi\|_{\infty}^{N+1}}{1-\|\varphi\|_{\infty}}
$$




\section{Conclusions}

We have introduced two slice regular compositions, which leads to the theory of slice composition operators. In particular, we established the Denjoy-Wolff type theorem about the dynamical behaviors of the iterates and the Littlewood subordination principle for slice regular functions. The slice regular theory is believed to be based upon two kind of operators, i.e., the slice regular product and slice regular compositions.

Acknowledgment This work was supported by the NNSF of China (11371337), RFDP (20123402110068).

\section{REFERENCES}

1. Alpay D., Colombo F., Sabadini I.: Pontryagin-de Branges-Rovnyak spaces of slice hyperholomorphic functions. J. Anal. Math. 121, 87-125 (2013)

2. Bourdon P. S., Fry E. E., Hammond C., Spofford C. H.: Norms of linear-fractional composition operators. Trans. Amer. Math. Soc. 356(6), 2459-2480 (2004)

3. Chaumat J., Chollet A.: On composite formal power series. Trans. Amer. Math. Soc. 353(4), 1691-1703(2001)

4. Colombo F., Gentili G., Sabadini I., Struppa D. C.: Extension results for slice regular functions of a quaternionic variable. Adv. Math., 222(5), 1793-1808 (2009)

5. Colombo F., Gentili G., Sabadini I.: A Cauchy kernel for slice regular functions. Ann. Global Anal. Geom. 37(4) , 361-378 (2010)

6. Colombo F., Gonzlez-Cervantes J. O., Sabadini I.: A nonconstant coefficients differential operator associated to slice monogenic functions. Trans. Amer. Math. Soc. 365(1), 303-318 (2013)

7. Colombo F., Sabadini I., Struppa D. C.: Noncommutative functional calculus. Theory and Applications of Slice Hyperholomorphic Functions. Progress in Mathematics, vol. 289, Birkhäuser/Springer, Basel, 2011.

8. Cowen C. C., MacCluer B. D.: Composition Operators on Spaces of Analytic Functions. Studies in Advanced Mathematics, CRC Press, Boca Raton, 1995.

9. de Fabritiis C., Gentili G., Sarfatti G.: Quaternionic Hardy spaces. Preprint 2013, www.math.unifi.it/users/sarfatti/Hardy.

10. Gallardo-Gutiárrez E. A., Partington J. R.: Norms of composition operators on weighted Hardy spaces. Israel J. Math. 196, 273-283(2013).

11. Gan X., Knox N.: On composition of formal power series. Int. J. Math. Math. Sci. 30(12), $761-770(2002)$

12. Gentili G., Stoppato C.: Zeros of regular functions and polynomials of a quaternionic variable. Mich. Math. J. 56(3), 655-667 (2008)

13. Gentili G., Stoppato C.: The zero sets of slice regular functions and the open mapping theorem. in Hypercomplex Analysis and Applications, ed. by I. Sabadini, F. Sommen. Trends in Mathematics (Birkhäuser, Basel, 2011),pp. 95-107.

14. Gentili G., Stoppato C., Struppa D. C.: Regular functions of a quaternionic variable. Springer Monographs in Mathematics, Springer, Berlin-Heidelberg, 2013.

15. Gentili G., Stoppato C., Struppa D. C., Vlacci F.: Recent developments for regular functions of a hypercomplex variable, in Hypercomplex Analysis, ed. by I. Sabadini, M. Shapiro, F. Sommen. Trends in Mathematics (Birkhäuser, Basel, 2009), 165-186.

16. Gentili G., Struppa D. C.: A new approach to Cullen-regular functions of a quaternionic variable. C. R. Math. Acad. Sci. Paris, 342(10), 741-744 (2006)

17. Gentili G., Struppa D. C.: A new theory of regular functions of a quaternionic variable. Adv. Math. 216(1), 279-301 (2007)

18. Gentili G., Struppa D. C.: Regular functions on the space of Cayley numbers. Rocky Mt. J. Math. 40(1), 225-241 (2010)

19. Gentili G., Vlacci F.: On fixed points of regular Möbius transformations over quaternions. Complex analysis and dynamical systems IV. Part 1, 75-82, Contemp. Math., 553, Amer. Math. Soc. Providence, RI, 2011. 
20. Ghiloni R., Perotti A.: Slice regular functions on real alternative algebras. Adv. Math. 226(2), 1662-1691 (2011)

21. Ren G. B., Wang X. P.: The growth and distortion theorems for slice regular functions. submitted.

22. Rocchetta C. D., Gentili G., Sarfatti G.: The Bohr theorem for slice regular functions. Math. Nachr. 285(17-18), 2093-2105 (2012)

23. Sarfatti G.: Elements of function theory in the unit ball of quaternions. Ph.D. Thesis, Università di Firenze, 2013.

24. Schimming R., Rida S. Z.: Non commutative Bell polynomials. Int. J. Algebra Comput. 6(5), 635-644 (1996)

25. Stoppato C.: Regular Möbius transformations of the space of quaternions. Ann. Global Anal. Geom. 39(4), 387-401 (2010)

26. Vlacci F.: Regular composition for slice-regular functions of quaternionic variable. in Advances in Hypercomplex Analysis, ed. by G. Gentili, I. Sabadini, M. V. Shapiro, F. Sommen, D. C. Struppa, Springer INdAM Series, Springer, Milan, 2013, pp. 141-147.

Guangbin Ren, Department of Mathematics, University of Science and Technology of China, Hefei 230026, China

E-mail address: rengb@ustc.edu.cn

Xieping Wang, Department of Mathematics, University of Science and Technology of China, Hefei 230026, China

E-mail address: pwx@mail.ustc.edu.cn 